Notfall Rettungsmed 2015 · 18:1016-1034 DOI 10.1007/s10049-015-0092-y

Online publiziert: 12. November 2015

(C) European Resuscitation Council (ERC),

German Resuscitation Council (GRC), Austrian Resuscitation Council (ARC) 2015
R. Greif ${ }^{1}$ A.S. Lockey ${ }^{2} \cdot$ P. Conaghan 3 - A. Lippert ${ }^{4} \cdot$ W. De Vries ${ }^{5} \cdot$ K.G. Monsieurs ${ }^{6,7}$

${ }^{1}$ Department of Anaesthesiology and Pain Medicine, University

Hospital Bern, University of Bern, Bern, Schweiz

${ }^{2}$ Emergency Department, Calderdale Royal Hospital, Halifax HX3 OPW, Salterhebble, UK

${ }^{3}$ School of Nursing, Midwifery \& Social Work, The University of Manchester, Manchester, UK

${ }^{4}$ Danish Institute for Medical Simulation, Center for HR, Capital Region of Denmark, Dänemark

${ }^{5}$ Knowledge Centre, ACM Training Centre, Elburg, Niederlande

${ }^{6}$ Emergency Medicine, Faculty of Medicine and Health Sciences, University of Antwerp, Antwerp, Belgien

${ }^{7}$ Faculty of Medicine and Health Sciences, University of Ghent, Ghent, Belgien

\section{Ausbildung und Implementierung der Reanimation}

\section{Kapitel 10 der Leitlinien zur Reanimation 2015 des European Resuscitation Council}

\section{Einführung}

Die Überlebenskette [1] wurde zur Formel des Überlebens [2] ausgebaut, denn das erklärte Ziel, mehr Leben zu retten, hängt nicht allein von solider, qualitativ hochwertiger Forschung ab, sondern auch von einer effektiven Ausbildung der Laien und professionellen Helfer [3]. Letztendlich müssen dort, wo Patienten mit Kreislaufstillständen behandeln werden, ressourceneffiziente Systeme implementiert werden, um das Überleben nach einem Kreislaufstillstand zu verbessern.

Dieses Kapitel beinhaltet die 17 ausbildungsrelevanten PICO-Fragen (Population, Intervention, Control (Kontrollintervention), Outcome (Ergebnis)), welche von der „Education, Implementation and Teams (EIT) Task Force" des International Liaison Committee on Resuscitation (ILCOR) von 2011 bis 2015 evaluiert wurden. Dieser Beurteilungs- und Evaluationprozess der gefundenen Evidenz folgte dem „Grading of Recommendations, Assessment, Development and Evaluation (GRADE)-Prozess“, der im ILCOR-Konsensus-Papier 2015 zur Wissenschaftlichkeit und den Trainingsempfehlungen („Consensus on Science and Training Recommendations 2015“ - CoSTR) publiziert wurde [4]. In dieser Publikation werden die neuen Empfehlungen zur Ausbildung und deren Implementierung zusammengefasst. zu diesem Kapitel haben beigetragen: John H.W. Ballance: Woolhope, Hereforddhire, UK

Alessandro Barelli: Teaching Hospital Agostino Gemelli, Rome, Italy

Dominique Biarent: Paediatric Intensive Care and Emergency Department, Hôpital Universitaire des Enfants, Université Libre de Bruxelles, Belgium

Leo Bossaert: University of Antwerp, Antwerp, Belgium

Maaret Castrén: Department of Emergency Medicine and Services, Helsinki University Hospital and Helsinki University, Finland

Anthony J. Handley: Hillcrest Cottage, Hadstock, Cambridge, UK
Carsten Lott: Department of Anesthesiology, University Medical Center, Johannes GutenbergUniversity, Mainz, Germany

Ian Maconochie: Paediatric Emergency Medicine, Imperial College Healthcare NHS Trust and BRC Imperial NIHR Grant holder, Imperial College London, UK

Jerry P. Nolan: Department of Anaesthesia and Intensive Care Medicine, Royal United Hospital, Bath UK and Bristol University, UK

Gavin Perkins: Warwick Medical School, University of Warwick, Coventry, UK, Critical Care Unit, Heart of England NHS Foundation Trust, Birmingham, UK

Violetta Raffay: Municipal Institute for Emergency Medicine Novi Sad, Novi Sad, Serbia
Charlotte Ringsted: Faculty of Health, Aarhus University, Denmark

Jasmeet Soar: Anaesthesia and Intensive Care Medicine, Southmead Hospital, Bristol, UK

Joachim Schlieber: Trauma Hospital Salzburg, Austria

Patrick Van de Voorde University Hospital and University Ghent, Belgium; Federal Department Health, Belgium:

Jonathan Wyllie: James Cook University Hospital, Middlesbrough, UK

David Zideman; Imperial College Healthcare NHS Trust, London, UK

Die Leitlinienverfasser bedanken sich bei Sam Richmond, der kürzlich verstarb, für seinen bedeutenden Beitrag zu diesem Kapitel. 
Dieses Kapitel beschreibt die Prinzipien des Unterrichts und Trainings in den lebensrettenden Basismaßnahmen (BLS) und erweiterten Cardiopulmonary-Resuscitation(CPR)-Maßnahmen. Eine deutliche Fokussierung erfährt der Unterricht der nicht technischen Fertigkeiten („nontechnical skills“, NTS), wie Kommunikation, Team- und Führungsverhalten. Darüber hinaus beinhaltet das Kapitel das ERC-Portfolio der Kurse und endet mit einem Ausblick auf Forschungsfragen im Lehrbereich und auf mögliche Entwicklungen der ERC-Kurse (ERC European Resuscitation Council).

Gründe für die verspätete Implementierung der letzten Leitlinien waren Verzögerungen in der Bereitstellung von Schulungsmaterialien und Probleme mit der Freistellung von Personal für CPRTrainings [5-7]. Um die Verbreitung der Leitlinien der Reanimation 2015 zu erleichtern und zeitgerecht durchzuführen, plante der ERC sorgfältig und frühzeitig die Übersetzungen in die europäischen Sprachen und die Verbreitung der neuen Leitlinien sowie des Schulungsmaterials für alle Kursformate. Dieses Kapitel liefert die Grundlage für erfolgreiche Unterrichtsstrategien, welche die Ausbildung in CPR verbessern werden.

\section{Zusammenfassung der \\ Änderungen seit den \\ ERC-Leitlinien 2010}

Es folgt eine Zusammenfassung der wichtigsten neuen Bewertungen oder Änderungen in den Empfehlungen für das Training, die Implementierung und die Teams seit den ERC-Leitlinien 2010:

\section{Training}

- Simulationspuppen mit großer Realitätsnähe („High Fidelity“) werden von den Lernenden sehr geschätzt, sind aber deutlich teurer als Standardreanimationspuppen („Lower Fidelity"). Es wird Zentren empfohlen, diese High-Fidelity-Simulationspuppen einzusetzen, wenn die Ressourcen zu Anschaffung und Unterhalt vorhanden sind. Die Verwendung von Lower-Fidelity-Reanimationspuppen ist aber für alle Niveaus der ERC-CPRKurse adäquat.
- CPR-Feedback-Geräte, die Anweisungen geben, sind sinnvoll, um die Kompressionsfrequenz und -tiefe, die Entlastung und die Platzierung der Hände zu verbessern. Geräte, die nur Töne abgeben, verbessern nur die Kompressionsfrequenz. Da sich die Helfer dabei auf die Frequenz konzentrieren, wirkt sich dies verschlechternd auf die Kompressionstiefe aus. Zurzeit gibt es keine Evidenz, die belegt, dass eine Ausbildung mit diesen Töne abgebenden Geräten im ERCKurs tatsächlich im Ernstfall zu verbesserten Überlebensraten bei den Patienten führt.

- Die Intervalle für Wiederholungstrainings werden je nach Kursteilnehmern (z. B. Laien- oder professioneller Helfer) unterschiedlich sein. Bekannterweise verschlechtern sich die CPR-Fertigkeiten bereits innerhalb von Monaten nach dem Training. Aus diesem Grund sind Strategien mit jährlichen Wiederholungstrainings möglicherweise nicht häufig genug. Auch wenn das optimale Intervall nicht klar ist, scheinen häufigere „niedrig dosierte“ Wiederholungstrainings eine erfolgreiche Strategie zu sein.

- Training in nicht technischen Fertigkeiten, wie Kommunikation, Teamführung und die Aufgabe des Einzelnen im Team, sind eine essenzielle Ergänzung zum Training der technischen Fertigkeiten. Derartige Schulungen sollen in alle „Life-Support“Kurse integriert werden.

- Leitstellenmitarbeiter spielen eine entscheidende Rolle in der Anleitung zur Laien-CPR. Um einem Laien in einer stressvollen Situation effizient und klar CPR-Anweisungen geben zu können, benötigen sie hierfür ein spezifisches Training.

\section{Implementierung}

- Das Debriefing, welches reale Reanimationsdaten miteinbezieht und auf die geleisteten Reanimationsmaßnahmen fokussiert, zeigte klare Verbesserungen bei den Reanimationsteams. Es gibt eine deutliche Empfehlung, Debriefings für Reanimationsteams durchzuführen.
- Regionale Versorgungssysteme einschließlich „Cardiac-Arrest“-Zentren (Zentren zur Behandlung von Kreislaufstillständen) sind zu unterstützen. Sie stehen in Zusammenhang mit gesteigertem Überleben und verbessertem neurologischem Status bei Patienten nach außerklinischen Kreislaufstillständen.

- Die Verwendung von innovativen Technologien und sozialen Netzwerken informiert Ersthelfer frühzeitiger und hilft ihnen, außerklinische Kreislaufpatienten und den nächstgelegenen AED schneller zu erreichen. Jegliche Technologie, die dafür sorgt, dass Ersthelfer früher mit der CPR, inklusive AED-Anwendung, beginnen können ist zu unterstützen.

- „Es bedarf eines Systems, um Leben zu retten“ (http://www.resuscitationacademy.com/). Einrichtungen (Rettungs-/Ambulanzorganisationen, „Cardiac Arrest“-Zentren), die Verantwortung in der Gesundheitsversorgung haben und Patienten im Kreislaufstillstand managen, müssen ihre Prozesse so evaluieren, dass sichergestellt wird, dass die zur Verfügung gestellte Behandlung das beste und höchste Maß an Überleben bietet.

\section{Trainingsstrategien für die Basismaßnahmen}

\section{Wer ist zu trainieren?}

BLS ist der Eckpfeiler der Reanimation. Es ist allgemein anerkannt, dass Ersthelfer-CPR für das Überleben von Kreislaufstillständen außerhalb des Krankenhauses entscheidend ist. Thoraxkompressionen und frühe Defibrillation sind die Hauptdeterminanten des Überlebens beim außerklinischen Kreislaufstillstand, und es gibt Hinweise, dass sich mit Einführung von Laientrainings das 30-Tageund 1-Jahres-Überleben verbessert hat [8, 9].

Aus diesen Gründen ist das primäre Ziel der Reanimationsausbildung das Training von Laien in CPR. Die Evidenz zeigt, das BLS-Laientraining die Anzahl der Ersthelfer erhöht, die in einer realen Situation BLS anwenden [10-12]. Der Be- 
griff „Laie“ ist weit gefasst und meint sowohl Menschen ohne jede formale Ausbildung in einem Gesundheitsberuf als auch solche, von denen CPR-Kompetenz erwartet wird (Rettungsschwimmer, Erste-Hilfe-Sanitäter oder Sport- und Sicherheitspersonal). Trotz des verbesserten Zugangs zu CPR-Laientraining gibt es beträchtliche Vorbehalte, die die Anwendung realer CPR verhindern. Die Gründe dafür sind vor allem: Angst vor Infektion, Angst, etwas falsch zu machen oder rechtlich belangt zu werden [13].

Wenn Familienmitglieder von Hochrisikopatienten in CPR trainiert werden, verringert das ihre Angst und die der Patienten, verbessert die emotionale Einstellung und verstärkt das Gefühl, CPR starten zu können und zu wollen, wenn es nötig wäre. In Hochrisikopopulationen (hohes Risiko für Kreislaufstillstand und niedrige Ersthelferrate) konnten spezifische Faktoren identifiziert werden, die zielgerichtetes Training, das auf die besonderen Bedürfnisse und Charakteristika der Betroffenen ausgerichtet ist, nahelegen $[14,15]$. Leider suchen mögliche Ersthelfer aus diesen Gemeinschaften von sich aus solche Trainings nicht, aber wenn sie sie besuchen, erlangen sie gute BLS-Fertigkeiten [16-18]. Es ist bei ihnen durchaus eine Bereitschaft vorhanden, sich trainieren zu lassen und die Erfahrungen mit anderen zu teilen $[16,17,19-21]$.

Die meiste Forschung über den Reanimationsunterricht wurde bei erwachsenen Helfern in Erwachsenen-CPR gemacht. Es ist anzunehmen, dass Kinder und Jugendliche andere Unterrichtsformen benötigen. Deshalb ist weitere Forschung notwendig, um die beste Methode für den BLS-Unterricht für Kinder und Jugendliche zu finden [22].

Einer der wichtigsten Schritte, um die Ersthelferrate und somit weltweit das Überleben nach Reanimation zu steigern, ist, Schulkinder zu unterrichten. Die American Heart Association machte sich schon 2011 für verpflichtendes Reanimationstraining an amerikanischen Schulen stark [23]. Davor zeigten Erfahrungen aus Seattle, das Unterricht von Schulkindern in den letzten 30 Jahren die Ersthelferrate und das Überleben signifikant steigerte. Von ähnlichen Steigerungsraten in CPR wird von skandinavischen Schulpro- grammen zur Reanimation berichtet [24]. Es würde reichen, Schulkinder ab dem 12. Lebensjahr $2 \mathrm{~h}$ pro Jahr in Wiederbelebungsmaßnahmen zu unterrichten [22]. Ab diesem Alter haben sie eine positive Haltung zum Erlernen solcher Fertigkeiten. Sowohl medizinisch professionelle Helfer wie auch die Schullehrer benötigen hierfür eine spezielle Schulung, damit sie das Potenzial der Kinder zum Erlernen von CPR voll ausschöpfen können [25]. Schulkinder und deren Lehrer sind Reanimationsmultiplikatoren sowohl im privaten Umfeld als auch in der Öffentlichkeit. Sie geben ihre erlernten CPR-Fähigkeiten an ihre Familienmitglieder weiter. So kann der Anteil an CPR-Trainierten in der Gesellschaft substanziell ansteigen, und langfristig führt das zu einem erheblichen Anstieg der CPR-Ersthelferrate [26].

Professionelle Helfer in allen Bereichen der Gesundheitsversorgung, von den Ambulanz- und Rettungsorganisationen über die allgemeinen Stationen der Krankenhäuser bis hin zu den Bereichen der Notfall- und Intensivmedizin müssen in CPR geschult werden. Unterbrechungen der Thoraxkompressionen tragen neben schlechter Thoraxkompression (inkorrekte Kompressionstiefe und -frequenz) wesentlich zu ineffektiver CPR bei [27]. Da schlecht ausgeführte CPR mit geringerem Überleben assoziiert ist, müssen diese Kernkomponenten in jedem CPR-Training besonders betont werden.

Gut geschultes und trainiertes Leitstellenpersonal ist in der Lage, Laien per Telefon in CPR anzuleiten und somit das Überleben von Patienten zu verbessern [28]. Die Schwierigkeit dabei ist, den Kreislaufstillstand zu erkennen, insbesondere wenn agonale Atmung vorliegt [29]. Das Training des Leitstellenpersonals muss sich auf die Bedeutung des Erkennens von agonaler Atmung und auch von Krampfanfällen als einem Aspekt von Kreislaufstillständen konzentrieren [30]. Spezielle Schulung braucht das Leitstellenpersonal in der Vermittlung der Anleitungen, mit denen sie die Ersthelfer in CPR instruieren [30].

\section{Wie ist zu trainieren?}

BLS-/AED-Ausbildungen sollen gezielt auf die Bedürfnisse der zu Schulenden ausgerichtet und so einfach wie möglich sein. Der immer leichtere Zugang zu den unterschiedlichsten Trainingsmethoden (digital, online, selbst gesteuertes Lernen, von Instruktoren gestalteter Unterricht) bietet verschiedene Alternativen des CPR-Unterrichts für Laien wie auch für professionelle Helfer. Die Effektivität dieser unterschiedlichen Möglichenkeiten des sog. integrierten Lernens („blended learning") ist leider noch unklar und muss hinsichtlich der Auswirkung direkt nach einem Kurs dringend erforscht werden. Letztendlich soll auch geklärt werden, ob solche CPR-Trainingsmethoden die Überlebensraten beim realen Kreislaufstillstand tatsächlich verbessern.

Das CPR-Training muss an die unterschiedlichen Bedürfnisse der Auszubildenden angepasst werden. Die Vielfalt der Unterrichtsmethoden ist notwendig, um sicherzustellen, dass CPR-Wissen und -Fertigkeiten erworben und behalten werden können. Dabei scheinen selbst instruierende Programme sowohl für Laien als auch für professionelle Helfer durchaus eine effiziente Alternative zu den klassischen, von Instruktoren geleiteten Kursen zu sein [31-35]. Es gibt sie mit oder ohne gleichzeitige praktische Übungen („hands-on practice“, wie Video, DVD, Online-Training oder direkt während des Trainings Feedback gebende Computer).

Helfer, von denen erwartet wird, dass sie $\mathrm{CPR}$ regelmäßig ausführen, müssen das Wissen den aktuellen Leitlinien entnehmen und dieses effektiv als Teil eines multiprofessionellen Teams anwenden können. Diese Helfer benötigen ein komplexeres Training, welches sowohl die technischen als auch die nicht technischen Fertigkeiten (Arbeiten im Team, Führungsverantwortung, strukturierte Kommunikation) beinhaltet $[36,37]$.

\section{Das BLS- und AED-Kursprogramm (Basismaßnahmen)}

Es gibt genug Evidenz, dass Laien nicht nur effektive CPR erlernen, sondern auch in der Anwendung eines AED unterrichtet werden können [38]. Die Einführung 
von in der Öffentlichkeit verfügbaren Defibrillatoren („public access defibrillator") demonstrierte, dass Laien effektiv defibrillieren können [39]. Die Frage, ob sie ein AED-Training brauchen oder ob die AEDs ohne weitere Erklärung verwendet werden können, ist noch nicht ausreichend beantwortet [40]. Ausbildungsprogramme für BLS/AED müssen an die Bedürfnisse der Auszubildenden angepasst und so einfach wie möglich gehalten werden. Egal welche Art des Unterrichts stattfindet, folgende Kernelemente müssen Bestandteile der BLS-/AED-Kurse sein:

- Die Bereitschaft, im Ernstfall mit CPR zu beginnen, sowie das Verstehen persönlicher bzw. umgebungsbedingter Risiken.

- Das Erkennen von Bewusstlosigkeit, Schnapp- oder agonaler Atmung bei nicht reagierenden Menschen, das Überprüfen von normaler Reaktion, das Öffnen der Atemwege und Überprüfen vorhandener Atmung, um den Kreislaufstillstand zu bestätigen [41, 42].

- Thoraxkompression von hoher Qualität (Beibehalten der Kompressionsfrequenz und -tiefe, vollkommene Entlastung zwischen den Kompressionen, minimale Zeiten ohne Kompression („hands-off time“) und externe Beatmung (Zeit und Volumen der Beatmung).

- Feedback, entweder durch Helfer während der CPR und/oder von Geräten, kann dazu beitragen, dass neu erworbene Fertigkeiten im Rahmen des BLS-Trainings besser behalten werden [43].

Unterricht von Standard-CPR vs. kontinuierliche Thoraxkompression

Die Rolle von Standard-CPR vs. CPR mit kontinuierlicher Thoraxkompression wird im BLS-Kapitel dieser ERC-Leitlinien diskutiert [42]. Um allen Mitbürgern ein CPR-Training schmackhaft zu machen, wird empfohlen, die Schulungsinhalte so einfach wie möglich zu vermitteln:

- Als Minimum sollen am Ende alle Bürger effektive Thoraxkompressionen durchführen können.
- Idealerweise erlernen alle die kompletten CPR-Fertigkeiten (Thoraxkompression und Beatmung im Verhältnis 30:2).

- Wenn die zur Verfügung stehenden Schulungszeiten eingeschränkt sind oder die Gegebenheiten des Unterrichts nichts anderes erlauben (z. B. eine durch die Leitstelle angeleitete CPR durch zufällig Anwesende, Massenveranstaltungen und öffentliche Kampagnen, im Internet verbreitete Videos), soll man sich auf CPR mit kontinuierlicher Thoraxkompression beschränken. Lokale Programme müssen Überlegungen zur Bevölkerungszusammensetzung vor Ort, den kulturellen Normen und der Einsatzhäufigkeit der Ersthelfer mit einbeziehen.

- Wer initial nur kontinuierliche Thoraxkompressionen gelernt hat, soll in der Folge auch in Beatmung unterrichtet werden. Idealerweise wird nach einer CPR-Schulung mit kontinuierlicher Thoraxkompression ein Standard-BLS-Training angeboten, welches Thoraxkompression und Beatmung in einer Schulungseinheit vermittelt.

- Laienhelfer mit Aufgaben der Fürsorge im weitesten Sinn (Erste-Hilfe-Personal, Sportstättenwarte oder Bademeister, Schul-, Heim- und Überwachungspersonal) müssen in StandardCPR (Thoraxkompression und Beatmung) geschult werden.

- Für die Reanimation von Kindern sollen Helfer lernen, das anzuwenden, was sie in CPR-Kursen über die Behandlung von Erwachsenen gelernt haben, da nichts zu tun auf jeden Fall das Schlechteste für das Überleben ist. Laien, die für Kinder verantwortlich sind (Eltern, Lehrer, Erzieher, Sporttrainer) und die Kinderreanimation erlernen wollen, können in modifizierten Erwachsenen-BLS-Kursen unterrichtet werden. Dieser Unterricht soll vermitteln, dass, bevor Hilfe geholt wird, 5 initiale Beatmungen, gefolgt von $1 \mathrm{~min}$ CPR, erfolgen sollen, wenn niemand zugegen ist, der alarmieren kann [44].

\section{BLS-/AED-Trainingsmethoden}

Es gibt eine Vielzahl von BLS-/AED-Trainingsmethoden für Laien und professionelle Helfer des Gesundheitswesens. Traditionell sind Kurse, die von Instruktoren geleitet werden, am häufigsten [45]. Eine sehr effiziente Alternative zu diesen Kursen sind gut geplante Programme zum Selbstlernen (z. B. Videos, DVDs, computerunterstütztes Feedback), welche durch kurze Anleitungen der Instruktoren unterstützt werden, v. a. zum Laientraining an den AEDs [18, 33, 34, 46-49].

Selbst wenn keine Instruktoren zur Verfügung stehen, sind diese Selbstlernprogramme eine akzeptable sowie pragmatische Lösung, um den Gebrauch eines AED zu schulen. Kurze Video-/Computer-Selbstlernprogramme mit minimaler oder ohne Instruktorenhilfe, die aber praktische Übungen mit dem AED anbieten („practice-while-you-watch“), können als effektive Alternative zu traditionellen Instruktoren-AED-Kursen betrachtet werden $[48,50,51]$.

Letztendlich ist bekannt, dass Ersthelfer AEDs auch ohne jegliches formales Training anwenden können. Aber nur das Vorhandensein eines AED in der Nähe ist keine Garantie dafür, dass dieser auch verwendet wird [52]. Der Vorteil des Trainings liegt sicherlich im Steigern des Bewusstseins über die Anwendung und den Nutzen von AEDs. Gleichzeitig können inkorrekte Mythen und Vorstellungen, wie der Irrglaube, damit Schaden anrichten zu können, ausgeräumt werden.

\section{Dauer und Frequenz von BLS-/ AED-Kursen mit Instruktoren}

Die optimale Dauer solcher BLS-/AEDTrainings, welche von Instruktoren geleitet werden, ist noch nicht gefunden worden. Diese wird sich eher nach den Besonderheiten der Teilnehmer (Laien, professionelle Helfer, Vortraining), dem Programm, dem Verhältnis von Instruktor zu Teilnehmern, der Möglichkeit zu praktischen Übungen und der Überprüfung der BLS-/AED-Kompetenzen am Ende des Kurses richten. Die meisten Studien zeigen, dass CPR-Fertigkeiten 3 bis 6 Monate nach dem Training rasch abnehmen [33, 
46, 53-55]. Dagegen werden AED-Kenntnisse etwas länger behalten $[56,57]$.

Obwohl es Hinweise gibt, dass häufiges und kurzes Auffrischungstraining möglicherweise das BLS-Training verstärkt und das Vergessen der Fertigkeiten etwas verlangsamt, sind mehr Studien zur Bestätigung erforderlich [53, 55-57].

Die gegenwärtige Evidenz zeigt, dass die Anwendung von AEDs (rasche und korrekte Platzierung der Elektroden) durch kurze Trainings von Laien und professionellen Helfern verbessert werden kann [49, 58-60]. Kurze KinderBLS-Auffrischungstrainings am Krankenbett von 2 min Dauer zeigten eine höhere CPR-Qualität in simulierten Reanimationen, egal wie trainiert wurde (Instruktor oder automatisches Feedback oder beides), [61] und verbesserte sich ständig mit jedem weiteren Training [62].

Ein Reanimationstraining, angeleitet von gleichgestellten Kollegen, ist ebenfalls eine sehr effektive BLS-Unterrichtsmethode. Diese Kollegen sind kompetente Trainer und Prüfer, oft in größerer Anzahl und zu geringeren Kosten verfügbar als klinisch eingesetzte Mitarbeiter. Auch studentische Instruktoren können Fertigkeiten zum Unterrichten, Prüfen und Bewerten, Organisieren und Forschen erwerben. Die Nachhaltigkeit solcher Programme ist durch die Planung der zukünftigen Instruktorenausbildung und eine konstante Führung möglich. Ein Überblick über 15 Jahre eines studentischen BLS-Kollegenprogramms an einer großen medizinischen Fakultät demonstrierte, dass die Teilnehmer mit dem Lernerfolg genauso zufrieden waren wie mit dem vorhergehenden auf Vorlesungen beruhenden Unterricht [63].

Wie gezeigt wurde, verbessert häufiges Training die CPR-Fertigkeiten, das Selbstvertrauen der Helfer und die Bereitschaft, eine CPR auszuführen. So wird empfohlen, dass jede Organisation und jeder Helfer die Notwendigkeit für häufigeres CPR-Wiederholungstraining prüfen soll, je nach Wahrscheinlichkeit für Kreislaufstillstände an deren Lebens- oder Arbeitsplatz. Ein Wiederholungstraining soll auf jeden Fall alle 12-24 Monate für jeden BLS-Kursbesucher stattfinden. Unter bestimmten Umständen sind zusätzlich häufigere, niedrig dosierte Auffrischun- gen oder Wiederholungen zu überlegen. Es wird aber empfohlen, dass Helfer die öfter mit Kreislaufstillständen konfrontiert werden, mit höherer Frequenz Auffrischungen besuchen sollen. Es ist bekannt, dass BLS-Fertigkeiten nach dem Training innerhalb von 3-12 Monaten abnehmen, [33, 46, 53, 54, 56, 64] dass häufiges Training die CPR-Fertigkeiten verbessert [34, 65-69] und das Selbstvertrauen der Helfer [65] wie auch ihre Bereitschaft, CPR auszuführen, stärkt [34].

\section{Die Verwendung von \\ CPR-Feedback-Geräten während des Trainings}

Für das Training von Laien und professionellen Helfern können CPR-FeedbackGeräte verwendet werden. Einige Geräte geben Signale, z. B. wie ein Metronom, um die Kompressionsfrequenz anzuzeigen, oder verbale Hinweise. Andere geben Feedback nach dem Ereignis, basierend auf den durchgeführten Aktionen (z. B. wird die Kompressionstiefe auf einem visuellen Display angezeigt), oder die Geräte bieten beiderlei Feedback. Wenn im Training solche Feedback-Geräte verwendet werden, können sich die CPR-Fertigkeiten verbessern [70]. Instruktoren und Helfer müssen sich bewusst sein, dass einige dieser Geräte die Kompressionstiefe bei komprimierbaren Patientenunterlagen (Matratzen) überschätzen [71, 72].

Eine systematische Bewertung der zur Verfügung stehenden Literatur über Studien an Puppen und Patienten zeigte, dass audiovisuelle Feedback-Geräte während der Reanimation die Helfer zu mehr leitlinienkonformen Thoraxkompressionen führen, aber es wurde kein Nachweis gefunden, dass dies zu einer Verbesserung des Patientenüberlebens führt [73]. Es wurden jedoch substanzielle Abweichungen in der Verbesserung der CPR-Leistung zwischen verschiedenen CPR-Feedback-Geräten entdeckt [74-76].

\section{Trainingsstrategien auf Fortgeschrittenen-Niveau}

Kurse in erweiterten lebensrettenden Maßnahmen („Advanced Life Support“, ALS) richten sich hauptsächlich an Personen, die im Gesundheitswesen arbeiten.
Ganz allgemein werden Wissen, Fertigkeiten und Haltungen abgedeckt, die notwendig sind, um als Mitglied oder Leiter in einem Reanimationsteam effizient $\mathrm{zu}$ funktionieren.

\section{Kursvorbereitung und alternative Strategien, um das CPR-Training zu verbessern}

Viele verschiedene Methoden können zur Vorbereitung der Teilnehmer an Kursen zu erweiterten lebensrettenden Maßnahmen herangezogen werden. Dies kann Lesestoff sein, in Form von Broschüren und/ oder e-Learning. Ein Test als Bestandteil der Vorbereitung vor dem Kurs verstärkt das Durcharbeiten dieser Unterlagen [7782]. Ein e-Learning-Programm mit CD vor dem ALS-Kurs wurde von den Teilnehmern geschätzt, da es ihr Verständnis der Kernelemente des ALS-Kurses verbesserte. Leider konnte aber keine Überlegenheit des Programms für kognitive und psychomotorische Fertigkeiten während standardisierten Reanimationssimulationen gefunden werden [83].

Unterschiedlich integrierte Lernmodelle („blended learning“), in denen z. B. elektronische Lehrmedien mit verkürzten instruktorengeleiteten Kursanteilen kombiniert werden, wurden als Pilotversuch für ALS-Kurse durchgeführt. Dies führte zu einer 5,7\% niedrigeren Bestehensrate in den CAS-Tests („Cardiac Arrest Scenario Test“ - die Überprüfung der Teilnehmerkompetenz, einen simulierten Kreislaufstillstand leitliniengetreu anzuleiten). Die Teilnehmer hatten ähnliche Bewertungen in der Wissensüberprüfung und den Fertigkeitseinschätzungen, und es gab keinen Unterschied im Bestehen bei Kursende, all dies bei Kostenreduktion um mehr als die Hälfte [84]. Dieses e-Learning-ALS-Kursformat wurde in Großbritannien flächendeckend eingeführt und an 27.170 Teilnehmern die Gleichwertigkeit zu den traditionellen, ausschließlich mit Instruktoren besetzten Kursen aufgezeigt [85]. Das Online-e-Learning-Programm von 6 bis $8 \mathrm{~h}$ Dauer wurde von den Teilnehmern vor dem modifizierten, durch Instruktoren angeleiteten eintägigen ALS-Kurs abgeschlossen. Die e-ALS-Testbewertungen waren im Multiple-Choice-Test vor und nach dem Kurs signifikant höher. Ebenso 
war die Bestehensquote beim ersten CASTest beim e-Learning-Kurs höher als beim Standardkurs, die Gesamtbestehensquote blieb aber gleich. Wenn die Vorteile in Bezug auf die gesteigerte Teilnehmerautonomie im Lernen, die bessere Kosteneffektivität, die geringere Arbeitsbelastung der Instruktoren und die verbesserte Standardisierung des Unterrichtsmaterials betrachtet werden, ermutigt dieser Bericht, solche e-Learning-Kurse für das CPRTraining weiter zu verbreiten.

\section{Die Prinzipien des Vermittelns von Fertigkeiten}

CPR-Fertigkeiten können schrittweise vermittelt werden, in dem ihre einzelnen Komponenten in Echtzeit gezeigt, die Fakten erklärt und von den Teilnehmern demonstriert werden. Das praktische Üben der Fertigkeiten soll die visuelle Vorstellung, das Verstehen und das kognitive Verarbeiten wie auch das Ausüben der Fertigkeiten fördern. Trotz des beschriebenen und bestechenden theoretischen Konzepts konnte keine Studie irgendeinen Vorteil verschiedener stufenweiser Zugänge zur Vermittlung der Fertigkeiten aufzeigen $[86,87]$.

\section{Die Grundlagen der Simulation im ALS-Kursunterricht}

Simulation ist ein integraler Bestandteil des Reanimationstrainings. Eine systematische Überblicksarbeit und Metaanalyse von 182 Studien, die 16.636 Teilnehmer von Reanimationsschulungen mit Simulationstrainings eingeschlossen hatten, zeigte verbessertes Wissen und Fertigkeitsausführung im Vergleich zu Trainings ohne Simulation [88].

Simulationstraining kann in einer Vielzahl von Situationen breit angewendet werden, vom Laien- und Ersthelfer bis zum Training ganzer Reanimationsteams inklusive deren Teamleiter. Somit können einzelne Teilnehmer, aber auch das Teamverhalten trainiert werden. Der essenzielle Teil dieser Lernform ist das an das Szenarientraining anschließende Debriefing mit einer kritischen Besprechung und Aufarbeitung des Erlebten, um weiteres Lernen zu ermöglichen.
Wenn nicht mit Schauspielpatienten gearbeitet wird, finden die meisten Simulationstrainings mit sehr realitätsnahen Simulationspuppen statt. Durch Computer werden die Vitalparameter, physiologische Daten und Befunde sowie Reaktionen auf Interventionen gesteuert. $\mathrm{Zu}$ sätzlich lassen sich an diesen Puppen Fertigkeiten ausführen und üben, wie Maskenbeatmung, Intubation und intravenöse oder intraossäre Gefäßzugänge [89]. Simulationstrainings an diesen sog. HighFidelity-Puppen scheinen denen an LowFidelity-Puppen im Hinblick auf die im Kurs erlernten Fertigkeiten leicht überlegen zu sein [90].

Diese High-Fidelity-Simulationspuppen sind wegen ihrer Realitätsnähe bei den Kursteilnehmern und Instruktoren beliebt, haben aber sehr hohe Anschaffungs- und Unterhaltungskosten. Es gibt keine Evidenz, dass die Teilnehmer in ERC-Kursen mehr oder besser lernen, wenn solche Puppen verwendet werden. Trotzdem sollen sie, wenn vorhanden, im CPR-Unterricht verwendet werden. Gibt es aber nur Low-Fidelity-Reanimationspuppen, sind auch diese ausreichend für Standard-ALS-Kurse.

Das Beibehalten der realen 2-min-Reanimationszyklen während der ALS-Simulation ist wesentlich, um das Szenario realistisch zu gestalten. Realität geht während des Übens verloren, wenn die Dauer der CPR-Zyklen willkürlich verkürzt wird, um eventuell mehr Szenarien üben $\mathrm{zu}$ lassen [91].

Viele der neuen Unterrichtsansätze sind vielversprechend, aber es benötigt mehr und bessere Forschung zu deren Effektivität, bevor diese Methoden dann in großem Stil eingesetzt werden können. Ein Beispiel dafür ist das Einbauen von Zusammenhängen wie „Aussage - Aktion“ in Kurse. Die Aussage „Es ist kein Puls vorhanden. Ich beginne mit Thoraxkompression “ mündet in die Aktion „Thoraxkompression“ [92]. Oder die sog. „Rapid Cycle Deliberate Practice“, welche Verbesserungen in den CPR-Fertigkeiten bei Kinderärzten in der Ausbildung brachte [93]. Nach einem Szenario mit Debriefing ohne Unterbrechung ist das Folgeszenario kürzer und wird an vorbestimmten Punkten kurz gestoppt, um di- rekt Feedback zu Handlungen oder Maßnahmen zu geben.

Das Training von nicht technischen

Fertigkeiten („non-technical

skills", NTS) inklusive Teamführung

und Teamtraining zur

Verbesserung der Reanimation

Die Ausführung einer erfolgreichen Reanimation ist in den meisten Fällen eine Teamleistung, und wie bei allen anderen Fertigkeiten, muss effektive Teamzusammenarbeit und das Führen eines Teams geübt werden [94, 95]. So führte die Implementierung eines Teamtrainingsprogramms zu einer verbesserten Krankenhausüberlebensrate nach Reanimation bei Kindern [96] sowie chirurgischen Patienten [97].

Die Schulung in der Simulation von nicht technischen Fertigkeiten (effektive Kommunikation, Situationsbewusstsein, Verhalten als Teammitglied und -leiter) und die Verwendung von Prinzipien aus dem „Crisis Ressource Management" konnte zeigen, das Erlerntes aus diesen Trainings in die klinische Praxis übertragen werden kann $[98,99]$. Die Leistung von Reanimationsteams verbessert sich in realen Reanimationen, aber auch in simulierten ALS-Krankenhausszenarien, wenn spezielle Team- oder Führungstrainings in die ALS-Kurse integriert werden [100-104]. Wenn diese Trainings so nahe wie möglich an der Arbeitsrealität der Teilnehmer liegen, können diese Teamzusammenarbeitskonzepte auch auf dem Niveau des Einzelnen besprochen werden $[105,106]$.

Spezifische Teamtrainings steigern die Leistungen des Teams, das Führungsverhalten und die Aufgabenbewältigung, und diese Effekte können bis zu $1 \mathrm{Jahr}$ anhalten [94, 95, 100, 101, 107-111]. Führungsverhalten allein neben den CPR-Fertigkeiten zu trainieren verbessert die CPR-Fertigkeiten nicht [112].

Instrumente zur Bewertung (hauptsächlich Checklisten) wurden entwickelt, validiert und sind empfohlen für individuelle Teammitglieder. Existierende Bewertungsskalen für Teamleistungen können auch gut für das Feedback an das Team verwendet werden [113-116]. 
Trainingsintervalle und die Beurteilung von Kompetenzen

Leider existiert wenig Evidenz über das Behalten des ALS-Wissens nach den Kursen [117]. Es wird angenommen, dass Teilnehmer mit mehr klinischer Erfahrung besser ihr Wissen und ihre Fertigkeiten über längere Zeit memorieren [118, 119]. Die schriftlichen ALS-Kurstests sagen wenig über die praktische Durchführung von Fertigkeiten aus und dürfen nicht als Ersatz für die Demonstration dieser klinisch relevanten Fertigkeiten verwendet werden [120, 121]. Die Prüfung am Ende des Trainings scheint einen positiven Effekt auf spätere Leistungen und die Dauer des Behaltens zu haben $[122,123]$.

Häufige Wiederholungstrainings am Arbeitsplatz in kleinen Dosen an den CPR-Puppen sparen Kosten, reduzieren die totale Trainingszeit und scheinen auch von den Lernenden bevorzugt zu werden $[124,125]$. Wiederholungstrainings sind ohne Zweifel notwendig, um das Wissen und die Fertigkeiten zu behalten, aber die optimale Frequenz für solche Auffrischungskurse ist unklar [124, 126-128].

Ein simulationsunterstütztes Auffrischungsprogramm 9 Monate nach neonatologischen Reanimationstrainings zeigte 15 Monate später verbesserte Leistungen in den Fertigkeiten und bessere Teamzusammenarbeit [129]. Das Teamverhalten wurde weiter verbessert, wenn die Assistenten in klinische Reanimationen eingebunden wurden oder freie Übungsmöglichkeiten am Simulator hatten.

\section{Die Verwendung von \\ Checklisten, Feedback-Geräten und Vor-Ort-Training}

Kognitive Hilfsmittel wie Checklisten können sowohl während der Simulation [130] als auch bei realen Patienten im Kreislaufstillstand [131] ein leitlinienkonformes Vorgehen verbessern, solange diese nicht den Start der Reanimation verzögern und die korrekte Checkliste verwendet wird. So verbesserte das Einführen von „Advanced-Trauma-Life-Support“Checklisten die Durchführung der Aufgaben gemäß den dort gegebenen Empfehlungen. Die Aufgaben wurden schneller und vollständiger ausgeführt [132].
Geräte mit direktem Feedback zu Kompressionsrate und -tiefe, Entlastung und Platzierung der Hände können während des Trainings herangezogen werden. Mit ihnen waren am Ende des Kurses die Fertigkeiten besser erlernt worden [61, 74, 76, 133-137]. Geräte, die nur Töne abgeben (Musik oder Metronom), können die Kompressions frequenz während des Trainings verbessern. Dies kann aber auf Kosten der Kompressionstiefe gehen, da vermehrt auf das Frequenzsignal geachtet wird [137-139]. CPR-Feedback-Geräte sorgen dafür, dass BLS-Fertigkeiten besser erlernt und behalten werden. Sie können ebenso in ALS-Kursen verwendet werden, um dort leitliniengetreue BLS-Leistungen zu gewährleisten. Trotz alledem soll die Verwendung dieser Feedback-Geräte nicht eine isolierte Intervention, sondern Teil eines breit angelegten Interventionskonzepts sein, welches auf umfassende Verbesserung der CPR-Qualität zielt [140].

Vor-Ort-Simulation bietet die Gelegenheit, ganze Teams zu trainieren [141] und gewährt Einblicke in Arbeitsprozesse auf Organisationsniveau [142]. Solche „On-site“-Teamtrainings lassen es eher zu, Teammitglieder aus verschiedenen Disziplinen und Berufsgruppen zu schulen. Dies kann ALS-Wissen [143] und -Fertigkeiten [144], Vertrauen und Vorbereitung [141] und das Vertrautsein mit der Umgebung [145] verbessern sowie verbreitete System- und Anwenderfehler identifizieren [142, 146, 147].

\section{Briefing und Debriefing nach Reanimationssimulation}

Das Debriefing nach Simulation im Rahmen des CPR-Unterrichts ist ein essenzieller Bestandteil des Lernprozesses. Im Gegensatz zum Szenario-Training ohne Debriefing, erfolgen während des Debriefings Reflexion und Lernen [148]. Das ideale Debriefing-Format ist noch nicht geklärt. Es gibt auch keinen Unterschied, ob zum Debriefing Videos verwendet werden oder nicht $[149,150]$.

\section{Implementierung und das Management der Änderungen}

Die „Formel des Überlebens“ endet mit der lokalen Implementierung [2]. Die
Kombination aus medizinischer Wissenschaft und effizienter Lehre reicht nicht aus, um Überleben zu verbessern, wenn die erforderlichen Maßnahmen dazu nicht getroffen und umgesetzt werden. Häufig ist auch Veränderungsbereitschaft erforderlich, um neue Visionen in eine lokale Struktur einzubetten. Um lang anhaltende Lösungen umzusetzen, sind oft schnelle und einfach erscheinende Schritte weniger zielführend, und oft sind Verhandlungsfähigkeit und Diplomatie notwendig. Ein erstklassiges Beispiel dafür ist das Einführen von CPR-Unterricht in Schulen. In den Länder, in denen das erreicht wurde, waren oft jahrelange Kampagnen und Überzeugungsarbeit notwendig, um die Gesetzgeber von diesen notwendigen Veränderungen zu überzeugen. Solche Modifikationen können von unten nach oben vorangetrieben werden, um aber erfolgreich zu sein, ist die Überzeugungsarbeit auch von oben nach unten anzustreben.

Dieses Kapitel war in den ERC-Leitlinien 2010 nicht enthalten und wurde aufgrund der Bedeutung für das Streben nach Verbesserung des Überlebens neu hinzugefügt.

\section{Einfluss der Leitlinien}

Die Einführung der Leitlinien basiert in jedem Land auf den international anerkannten Leitlinien zur kardiopulmonalen Reanimation. Die nationalen Lehrstrategien sind daher abhängig von den evidenzbasierten Aussagen zum Management der Kreislaufstillstands. Daher lautet die wichtigste Frage, ob diese Leitlinien zu sinnvollen und verbesserten Reanimationsergebnissen führen.

Die Autoren räumen hier bereitwillig ihren Interessenskonflikt ein - wenn wir uns eingestehen müssen, dass unsere Leitlinien keinen messbaren Vorteil erbringen, müssen wir den Aufwand infrage stellen, diese erarbeitet zu haben. Die gefundene Evidenz lässt einen positiven Nutzen erkennen, sofern man das Überleben bis zur Krankenhausentlassung [8, 151-156], das Wiedereinsetzen des Spontankreislaufs [8, 151-155] und die Ausführung der Reanimation $[8,153]$ betrachtet. Abgesehen davon ist die Wahrscheinlichkeit eines Nutzens höher als ein möglicher Schaden. 


\section{Reanimationszentren (",cardiac arrest centres")}

In den letzten Jahren haben regionale Gesundheitssysteme die Behandlung von Schlaganfall, Polytrauma und Herzinfarkt vorangetrieben. Dies wurde zumeist durch die Zentralisierung begrenzter Ressourcen erreicht und weniger durch Evidenz aus randomisierten Studien. Es gibt zunehmende Hinweise, dass der Transport von Patienten mit einem außerklinischen Kreislaufstillstand in ein spezialisiertes Reanimationszentrum mit einem erhöhten neurologisch unbeeinträchtigten Überleben verbunden sein könnte [157-170]. Die gegenwärtig verfügbaren Studien ergeben keine einheitlichen Aussagen bezüglich spezifischer Faktoren, welche zu einem verbesserten Behandlungsergebnis beitragen. Weitere wissenschaftliche Untersuchungen müssen durchgeführt werden, um die spezifischen Aspekte von Reanimationszentren aufzuzeigen, die verbessertes Überleben bringen können. Genauso muss der Einfluss der Transportzeit und die Frage, ob Sekundärtransporte in solche Zentren die gleichen Vorteile erbringen, untersucht werden.

Szenarienbasiertes Simulationstraining und dessen Auffrischung, regelmäBiges Üben im Team, v. a. bezüglich der Platzierung der Ausrüstung, sind für das Personal in Herzkatheterlabors notwendig. Im Rahmen der Einführung von mechanischen Reanimationshilfen in den klinischen Alltag konnte eine signifikante Lernkurve beobachtet werden [171]. Bei länger andauernden Reanimationsmaßnahmen im Herzkatheterlabor konnte durch die Einführung einer strukturierten Herangehensweise die Zusammenarbeit des Teams verbessert werden [172].

\section{Nutzung der aktuellen Informationstechnologie und der sozialen Medien}

Die weite Verbreitung von Smartphones und Tablets führte zur Entwicklung vieler Möglichkeiten zur Nutzung von Apps und sozialen Medien. Diese lassen sich in folgende Kategorien unterscheiden:
1. Einfache Informationsbeschaffung: Apps, welche die Reanimationsalgorithmen anzeigen.

2. Interaktive Informationsbereitstellung: Apps, welche den aktuellen Standort benutzen können, um den nächstgelegenen AED anzuzeigen.

3. Interaktive Lehrmöglichkeiten: interaktive Apps, welche den Nutzer in das Lernen einbinden, indem er oder sie sich aktiv mit dem Lehrangebot auseinandersetzen müssen (z. B. Lifesaver; www.life-saver.org.uk).

4. Gemischte Lernkonzepte für Wiederbelebungskurse: Hier konnte gezeigt werden, dass ein e-Learning-Programm, verbunden mit verkürztem instruktorgeleitetem Training, einem konventionellen erweiterten Wiederbelebungskurs (ALS) ebenbürtig ist [85].

5. Feedbacksysteme: Die Nutzung eines Accelerometers in Echtzeit verbessert die Frequenz und Tiefe der Kompressionen und kann Daten für die Nachbesprechung (Debriefing) speichern.

6. Benachrichtigung und Alarmierung von Ersthelfern: Die Nutzung eines solchen Systems, bei dem freiwillig registrierte BLS-Trainierte zu Reanimationen in der näheren Umgebung geschickt werden können, lässt das therapiefreie Intervall im Vergleich zu den Eintreffzeiten des regulären Rettungsdienstes verkürzen $[174,175]$.

7. Nutzung der sozialen Medien, um Informationen, ggf. im Rahmen von Kampagnen, in einem breiteren Rahmen zu streuen.

Schließlich kann man sagen, dass die aktuellen Informationstechnologien sowie soziale Medien wirkungsvolle Mittel für die Verbreitung und Umsetzung sind. Ihre Weiterentwicklung und Nutzung soll gefördert und deren Effekt auf das Überleben analysiert werden.

\section{Messung der Effizienz von Reanimationssystemen}

Nachdem Systeme entwickelt wurden, die das Outcome nach einem Kreislaufstillstand verbessern, muss deren Einfluss genau untersucht werden. Dies ist besonders für größere Systeme mit multifakto- riellen Komponenten wichtig, bei denen diese einzeln oder in Kombination nützlich sein könnten. Beispielsweise ließ sich zeigen, dass weitere Untersuchungen zur Effektivität der Reanimationszentren notwendig sind.

Die Messung der Ausführung und Effizienz der CPR-Maßnahmen und die Einführung von Qualitätsmanagementsystemen werden das Erreichen optimaler Ergebnisse weiter fördern [102, 176-181].

\section{Debriefing nach Reanimationen im innerklinischen Bereich}

Vertiefte Nachbesprechungen des innerklinischen Reanimationsteams zu seiner Arbeitsweise nach einem realen Kreislaufstillstand (nicht im Training) kann zu einem verbesserten CPR-Ergebnis führen. Dies kann entweder in Echtzeit mit Aufzeichnung technischer Reanimationsparameter (beispielsweise durch die Aufzeichnung der Thoraxkompression) oder im Rahmen eines strukturierten, auf die Durchführungsqualität fokussierten Debriefings erfolgen $[102,182]$. Die ideale Herangehensweise an so ein Debriefing ist noch nicht geklärt, so wenig wie das ideale Intervall zwischen der Reanimation und dem Debriefing. Obwohl die Durchführung einer solchen Nachbesprechung auch für eine außerklinische Reanimationssituation intuitiv richtig erscheint, gibt es bisher keine Evidenz für oder gegen ihren Nutzen.

\section{Medizinische Notfallteams für Erwachsene}

Wenn man die Überlebenskette des Kreislaufstillstands betrachtet, steht am Anfang die Früherkennung des zunehmend kritisch kranken Patienten und das Verhindern des Kreislaufstillstands. Beachtlich viele Untersuchungen zur Rolle des medizinischen Notfallteams („,medical emergency team ", MET) wurden evaluiert. Wir empfehlen solche METs generell und deren Aufwertung im Speziellen (beispielsweise durch höhere Alarmierungsraten und sehr erfahrenes ärztliches Personal im Team), da damit niedrigere Zahlen von Atem-Kreislauf-Stillständen [183189] sowie höhere Überlebensraten [184, 186-190] verbunden sind. 
Wir empfehlen, dass solche Systeme:

1. Mitarbeiterschulungen über die Symptome eines sich rasant verschlechternden Patienten durchführen,

2. angemessenes und regelmäßiges Erheben der Vitalfunktionen der Patienten durchführen,

3. klare Handlungsanweisungen (beispielsweise durch Alarmierungskriterien oder ein Frühwarnsystem) beinhalten, um das Personal in der Früherkennung von sich verschlechternden Patienten zu unterstützen,

4. klare einheitliche Alarmierungswege für weitere Unterstützung vorhalten,

5. eine adäquate klinische Antwort auf solche Alarmierungen bieten.

\section{Training in Bereichen mit limitierten Ressourcen}

Es gibt unterschiedliche Unterrichtstechniken für die BLS- und ALS-Maßnahmen in Gegenden oder Systemen mit geringeren Ressourcen. Diese beinhalten Simulation, multimedialen Unterricht, selbst gesteuertes Lernen (auch am Computer) und Kurse mit eingeschränkt anwesenden Instruktoren. Einige dieser Techniken sind günstiger und benötigen weniger Ausbilder als traditionelle Unterrichtsformate. Manche Techniken tragen auch zur weiteren Verbreitung von ALSund BLS-Training bei. Die Empfehlung für solche Unterrichtsstrategien ist in diesen Bereichen mit geringeren Ressourcen begründbar, obwohl die optimale Strategie noch nicht geklärt ist und sich zudem noch zwischen den einzelnen Ländern unterscheidet [191-197].

\section{Training zu ethischen bzw. Fragen der Ersten Hilfe}

Kenntnisse zum Unterlassen/Beenden von Reanimationsmaßnahmen als Bestandteil der Schulungen für professionelle Helfer sowie der Umgang mit kürzlich Verstorbenen werden im Ethik-Kapitel der ERC-Leitlinien 2015 thematisiert [198]. Das Erste-Hilfe-Kapitel der ERCLeitlinien 2015 beinhaltet Hinweise zum Unterricht der Ersten Hilfe und zu deren Trainingsprogrammen auch im Rahmen von Kampagnen der öffentlichen Gesundheitsfürsorge [199].

\section{Das ERC-Kursprogramm}

Der ERC hat zahlreiche Kurskonzepte entwickelt, um unterschiedliche Anwender gezielt anzusprechen, von den Basisreanimationsmaßnahmen (BLS) für Laienhelfer bis zu erweiterten Maßnahmen (ALS) für professionelle Helfer aus den Gesundheitsberufen. ERC-Kurse vermitteln die notwendigen Kompetenzen, um CPR in klinischer Umgebung auf dem erwarteten Niveau auszuführen. Neben den eigentlichen Reanimationsmaßnahmen gilt es auch, den nicht technischen Fertigkeiten, dem Training der Leitungsfunktion, der Anwendung ethischer Prinzipien, den zeitgemäßen Unterrichtsstrategien sowie einer Organisationsentwicklung zunehmende Beachtung zu schenken, um das Überleben nach einem Kreislaufstillstand zu verbessern. In speziellen Kursen werden diese Kompetenzen unterrichtet, andere Kurse sind den Unterrichtsstrategien gewidmet.

Die ERC-Kurse sind auf Kleingruppenunterricht ausgerichtet mit einem hohen Instruktor-Teilnehmer-Verhältniss. Sie nutzen „blended learning“ und unterschiedliche Unterrichtsmethoden wie interaktive Diskussionen, Arbeitsgruppen, Übungen zu spezifischen Fertigkeiten sowie der Simulation mittels Reanimationspuppen werden verwendet [200, 201].

Aktuelle Informationen zu den ERCKursen sind unter den „ERC-Kursregularien“ auf der ERC-Internet-Seite verfügbar (https://www.erc.edu/index.php/doclibrary/en/). Die Kursregularien beschreiben im Detail die ERC-Terminologie und -Definitionen, die Besonderheiten der Organisation und des Managements der unterschiedlichen Kursformate und deren Qualitätskontrolle, die Aus- und Weiterbildung vom Instruktor bis zum Kursdirektor, von Instruktorenausbildern und ERC-Educatoren, die ERC-Prüfungsregularien zur Zertifizierung und Rezertifizierung sowie ERC-Richtlinien zu professionellen Verhaltensweisen bis hin zu Beschwerdeverfahren.

\section{Ethos}

Die ERC-Kursinstruktoren sind geschult, zu unterrichten und zu prüfen. Ziel ist es, ein unterstützendes, auf die Auszubilden- den zentriertes Klima zu schaffen, welches das Lernen (Verstehen von Wissen und Einprägen von Fertigkeiten) unterstützt. In der Teilnehmer- und Instruktorengruppe („faculty“) wird die Verwendung von Vornamen empfohlen, um Berührungsängste zu reduzieren. Die Interaktionen zwischen den Instruktoren und den Teilnehmern sind geprägt vom Interesse aneinander und von der Bereitschaft, von den Erfahrungen der jeweils anderen zu lernen. Einstellungs- und Verhaltensänderungen werden durch konstruktives und korrigierendes Feedback und in den Szenarien-Debriefings erreicht. Ein Mentorensystem soll helfen, die Teilnehmer mittels Feedback zu unterstützen. Insbesondere in den Prüfungssituationen ist Stress unvermeidbar [202], die Instruktoren sind jedoch angehalten, dem Teilnehmer zu ermöglichen, sein Bestes zu geben. Das übergeordnete Ziel der ERC-Kurse ist es, die Reanimationsleistungen zu verbessern und somit die Überlebensrate nach einem Kreislaufstillstand zu steigern.

\section{Kursverwaltung und Management}

Die ERC-Kurse werden vom sog. Joint International Course Committee (JICC) unter der Leitung des Board Director for Training and Education (DTE) geregelt. Das JICC setzt sich aus den Vorsitzenden der International Course Committees (ICC) für alle Kursformate zusammen [BLS/AED, Immediate Life Support (ILS), ALS, Neonatal Life Support (NLS), European Paediatric Immediate Life Support/ European Paediatric Advanced Live Support (EPILS/ EPALS), Generic Instructor Course (GIC)].

Auf nationaler Ebene haben die National Resuscitation Councils (NRC) nationale Kursdirektoren für jedes Kursformat ernannt.

Um die Teilnehmerverwaltung zu erleichtern, wurde ein webbasiertes Kursverwaltungsprogramm durch den ERC entwickelt (http://courses.erc.edu).

Die Teilnehmer können sich online anmelden, oder sie wenden sich direkt an den Kursorganisator, um ihr Interesse am Kurs zu bekunden.

Am Ende eines Kurses generiert das System nummerierte Zertifikate für die erfolgreichen Teilnehmer sowie für alle 
Instruktoren. Zur Qualitätssicherung ist ein Evaluierungswerkzeug für jeden Kurs verfügbar, dessen Resultate für die NRCs, die Nationalen Kursdirektoren (NCDs) und ICC-Mitglieder zugänglich ist. Erfolgreiche Teilnehmer werden als „Anwender" („Provider") klassifiziert.

\section{Sprache}

In der Aufbauphase wurden die ERCKurse durch eine internationale Instruktorengruppe in Englisch unterrichtet. Nachdem nun lokale Instruktoren ausgebildet und die Manuale und Kursunterlagen übersetzt wurden, können die NRCs die Kurse in der jeweiligen Landessprache anbieten. Dies darf aber nicht die Qualitätskontrolle der Kurse und die Instruktorenweiterbildung behindern. Ebenso ist es wichtig, dass die Übersetzung der neuen Leitlinien und des Kursmaterials die Implementierung dieser Leitlinien nicht verzögert [5].

\section{Instruktorenausbildung}

Teilnehmer, welche im Kurs durch eine überdurchschnittliche Leistung aufgefallen sind und zudem besondere Qualitäten in Gruppenleitung und Gruppenarbeit unter Beweis gestellt haben und die klinisch glaubwürdig erscheinen, sich entsprechend verbal ausdrücken können und darüber hinaus unterstützend und motivierend wirken, können durch die Faculty als „Instruktorenpotenzial“ (IP) bezeichnet werden. Teilnehmer mit einem IP aus irgendeinem Kursformat werden $\mathrm{zu}$ einem Generic Instructor Course (GIC) eingeladen. Teilnehmer mit Instruktorenpotenzial der BLS-/AED-Kurse werden zu einem eigenen BLS-/AED-Instruktorenkurs eingeladen.

Beim GIC ist für die Vermittlung der Lehrprinzipien des ERC ein sog. Educator verantwortlich, der sich einem spezifischen Training in Medizindidaktik und den Prinzipien der Erwachsenenbildung (ERC - Educator Master Class) unterzogen hat.

\section{Vom,Instructor Candidate" (IC) zum „Full Instructor" (FI)}

Nach erfolgreicher Teilnahme am GIC werden die IPs Instruktorenkandidaten und unterrichten normalerweise zwei Provider-Kurse unter Aufsicht der KursFaculty, welche konstruktives Feedback zur Unterrichtsleistung im Kurs gibt, mit dem Ziel, dem Instructor Candidate den Status eines Vollinstruktors (Full Instructor) zu verleihen. Diese Entwicklung vom IC zum FI ist im „The Learning Path to Become an ERC Instructor “ (https://www. erc.edu/index.php/doclibrary/en/225/1/) hinterlegt. Dieses Feedback-Instrument unterstützt das Erlernen der Unterrichtspraxis vom GIC über die ersten ICs und erlaubt es, spezifische Lernziele für die weiteren Lernschritte klar auszuformulieren.

\section{Kursdirektor(CD)-Status}

Ein anerkannter Kursdirektor kann jeden ERC-Kurs leiten. CDs werden von NCD vorgeschlagen und von ihrem NRC oder dem entsprechenden ICC anerkannt. CDs sind erfahrene Instruktoren, die klinisch glaubhaft sind, herausragende Qualitäten als Lehrer, Mentor und Prüfer gezeigt haben und die Fähigkeit besitzen, eine Instruktorengruppe zu leiten.

\section{Allgemeine ERC-Kursprinzipien (ERC-Kursregularien \\ auf www.erc.edu)}

\section{Inhalt von ERC-Kursen}

Alle ERC-Kurse folgen den aktuell gültigen ERC-Leitlinien. Jeder Kurs hat sein spezifisches Kurshandbuch (Manual) oder Unterrichtsheft, welches das erforderliche Vorwissen vor dem Kursbeginn bereitstellt. Die Teilnehmer erhalten das Manual im Vorfeld, um sich auf den jeweiligen Kurs vorzubereiten. Dazu gehört auch das Ausfüllen eines verbindlichen Multiple Choice Questionnaire (MCQ; ausgenommen für BLS/AED, ILS und EPILS), der erreichen soll, dass die Teilnehmer die Kursunterlagen vor ihrer Teilnahme am Kurs lesen.

Alle ERC-Kurse beinhalten interaktive Vorträge und Gruppendiskussionen, Kleingruppenarbeiten, praktischen Unterricht von Fertigkeiten und, für ein Training auf fortgeschrittenem Niveau, klinisch orientierte Reanimationssimulationen (CAS) und Notfallszenarien. Bei den meisten Kursformaten können Inst- ruktoren ihren Unterricht auf die lokalen Bedürfnisse der Teilnehmer anpassen.

\section{Immediate- und Advanced-Life-Support-Kurse}

Immediate- und Advanced-Life-SupportKurse haben das Ziel, Mitarbeiter im Gesundheitswesen zu schulen. Die KursCurricula haben Kerninhalte, können aber den individuellen Lernbedürfnissen der jeweiligen Patientenpopulation und der Rolle des Einzelnen im Behandlungssystem bei einem Kreislaufstillstand angepasst werden. Die Kerninhalte für diese Kurse beinhalten:

- Vorbeugen eines Kreislaufstillstands, [203, 204]

- Thoraxkompressionen von hoher Qualität (Einhaltung von Frequenz, Tiefe, vollständige Entlastung und Minimierung der Unterbrechungszeit) und Beatmung unter Nutzung von einfachen Hilfsmitteln (z. B. Taschenmaske, Beatmungsbeutel),

- Defibrillation (mit Laden während der Kompressionen) ohne Kontakt der Hände auf dem Brustkorb während der Defibrillation,

- Algorithmen der erweiterten Wiederbelebung und Medikamentengabe bei Kreislaufstillstand,

- Nicht technische Fertigkeiten (z. B. Führungsverhalten, Teamtraining und Kommunikation).

\section{Immediate-Life-Support-Kurse}

Die ILS-Kurse für Erwachsene und EPILSKurse für Kinder sind eintägig und fokussieren auf die Ursachen und Prävention von Kreislaufstillständen, die ABCDEHerangehensweise an den kritisch kranken Patienten, den Beginn effektiver BLS inklusive der Anwendung eines halbautomatischen externen Defibrillators (AED), dem Initiieren der Überlebenskette sowie der CPR-Basisfertigkeiten (z. B. effektive Thoraxkompressionen und sichere Durchführung einer Defibrillation, Basismaßnahmen der Atemwegssicherung, Maßnahmen bei Erstickungsanfällen, Legen intravenöser oder intraossärer Gefäßzugänge sowie Medikamentengabe beim Kreislaufstillstand) [205]. Diese Kurse sind einfach gehalten und auf Kleingruppenunterricht ausgelegt. Ziel ist dabei die Schulung der Teilnehmer an den Geräten 
(z. B. Defibrillator), welche in der Arbeitsumgebung der Teilnehmer vorhanden ist, sowie das Management der ersten Minuten eines Kreislaufstillstands, bis weitere professionelle Hilfe eintrifft.

\section{ALS-Kurse}

Die ALS Kurse für Erwachsene, EPALS für Frühgeborene und Kinder und NLS für Neugeborene bauen auf Wissen und Fertigkeiten der BLS- oder ILS-/EPILSKurse auf. Diese stellen die Grundlagen für die erweiterten Zweitageskurse mit Betonung auf der sicheren Defibrillation und der EKG-Interpretation, dem Management des Atemwegs, der Beatmung und dem Legen eines Gefäßzugangs, dem Vorgehen bei Peri-Arrest-Rhythmen und den speziellen Umständen in Bezug auf die Ursache der kritischen Erkrankung, Unfall und Kreislaufstillstand. Die Versorgung nach einer Reanimation, ethische Aspekte der Reanimation und das Verhalten gegenüber Hinterbliebenen sind ebenfalls Inhalte der ALS-Kurse. Diese sollen es dem Anwender ermöglichen, die erste Stunde bei kritischer Krankheit oder Unfall oder Kreislaufstillstand abzudecken. Sie sind nicht vorgesehen, erweiterte Intensivmedizin oder Kardiologie zu schulen.

\section{Das Treffen der Instruktorengruppe (,faculty meeting")}

Die Instruktoren treffen sich mit dem Kursdirektor, der dieses Treffen leitet, üblicherweise am Anfang und am Ende eines jeden Kurstages mit dem Ziel, die Lehrenden über den Kurs zu informieren und Leistungen und Fortschritte jedes Teilnehmers zu evaluieren und zu beurteilen. Beim letzten Treffen wird die Leistung jedes Kandidaten besprochen, um zu entscheiden, ob der Kurs erfolgreich abgeschlossen wurde und ob dieser Teilnehmer den Status als potenzieller Instruktor erhält. Die IC (Instruktorenkandidaten) des Kurses werden ebenfalls bezüglich ihrer Lehrleistung beurteilt. Diese Faculty-Meetings bieten außerdem die Möglichkeit eines Debriefings am Ende des Kurses.

\section{Beurteilung und Feedback}

Während des ganzen Kurses beurteilt die Faculty jeden Kandidaten formativ und individuell. Die Leistungen und die Einstellung der Kandidaten werden in den täglichen Faculty-Meetings diskutiert, was bei Bedarf in Feedback oder Mentoring münden kann. Die Instruktoren wurden in einem Rahmenwerk ausgebildet, das es ermöglicht, zeitnahes, konstruktives, ziel- und lernorientiertes Feedback zu geben, welches auf künftige Verbesserungen zielt. Dieses Erarbeiten eines Handlungsplans soll es den Lernenden ermöglichen, ihre gewünschten Ziele mit der Kursteilnahme zu erreichen.

Das ERC-Standard-Feedback-Format ist „Das Lehrgespräch“ („The Learning Conversation“ "https://www.erc.edu/ index.php/doclibrary/en/225/1/). Dieses Lehrgespräch beginnt mit der Einladung zu reflektieren und zielt in erster Linie auf die Themen, welche die Kandidaten von sich aus diskutieren möchten. Es folgt eine Diskussion über die Schlüsselmomente während der Simulation, über die der Ausbilder diskutieren möchte und in die die Beiträge der Gruppe und anderer Instruktoren eingebunden werden. Alles Wichtige zur Arbeitsweise und den Leistungen wird dann zusammengefasst mit dem Ziel, einen spezifischen Plan für den Kandidaten zu entwickeln, um dessen zukünftige Leistung zu verbessern.

Die Arbeitsweise der Kandidaten während der BLS-, ILS- und GIC-Kurse wird kontinuierlich beurteilt. Die Kompetenzen der Kandidaten werden an vorbestimmten Kriterien gemessen; für den Erhalt des Kurszertifikats ist jedoch keine summative Prüfung erforderlich.

Am Ende der NLS- und ALS-Kurse wird im Rahmen eines Abschlusstests in Form der Reanimationssimulation (CAST) überprüft, ob das erworbene Wissen und die erlernten praktischen Fertigkeiten angewendet werden können und ob er zur Leitung eines Reanimationsteams fähig ist. Die Verlässlichkeit sowie die Messeigenschaften dieser CAST wurden nachgewiesen [121, 206, 207]. Das Kernwissen wird mittels eines schriftlichen MCQ-Tests am Kursende abgefragt.

\section{Mentoring}

Das Mentoring ist ein essenzieller Teil aller ERC-Kurse und bietet den Teilneh- mern die Möglichkeit zu individuellen Gesprächen mit Kursinstruktoren als Rollenmodell und Bezugsperson. Im Kursverlauf finden regelmäßig Einzel- und Gruppenmentorengespräche statt.

\section{Spezielle CPR-Kursformate des ERC}

Basisreanimationkurse und AED-Kurse (BLS/AED) und der BLS-/AED-Instruktorenkurs

BLS-/AED-Kurse sind für alle Bürger geeignet, inklusive Laienhelfer und ausgebildete Ersthelfer (Erste-Hilfe-Personal, Rettungsschwimmer), Personen, die im weitesten Sinne Mitmenschen betreuen (Lehrer, Pflegedienstmitarbeiter, Sicherheitspersonal) sowie selbstverständlich alle klinischen und außerklinischen Mitarbeiter im Gesundheitswesen (Leitstellenmitarbeiter, Allgemeinmediziner, Zahnärzte, Medizinstudenten und Krankenpflegeschüler und alle anderen, die mit einem Kreislaufstillstand konfrontiert werden könnten). Es werden kombinierte BLS- und AED-Kurse empfohlen. Die BLS-/AED-Kurse haben das Ziel, dass alle Teilnehmer die Kompetenzen erlangen, einen Kreislaufstillstand zu erkennen, sofort mit effektiven Thoraxkompressionen zu beginnen, adäquate Hilfe zu rufen sowie einen AED sicher zu bedienen. Diese Kurse richten sich an Kinder sowie Erwachsene und unterrichten Kinder- und Erwachsenenreanimation.

Der ERC-BLS-/AED-Instruktorenkurs ermöglicht es Teilnehmern mit einem gültigen BLS-/AED-Zertifikat, welchen Instruktorenpotenzial zugesprochen wurde, die Tätigkeit eines BLS-/AED-Instruktors zu erlernen.

\section{Immediate-Life-Support(ILS)-Kurs}

Der ILS-Kurs ist für die Mehrheit der Mitarbeiter im Gesundheitswesen für alle Disziplinen und Berufe ausgerichtet, die mit einem Erwachsenen im Kreislaufstillstand zwar selten konfrontiert sind, aber dennoch potenzielle Ersthelfer oder Mitglieder eines Reanimationsteams sein könnten [208]. Die angewendeten ILSKompetenzen führen zu suffizienten Reanimationsmaßnahmen in den ersten Minuten bis zur Übernahme durch das Reanimationsteam [209]. In einer Kohortenstudie ließ sich nach der Einführung 
eines ILS-Programms eine geringere Zahl an Reanimationsalarmen bei tatsächlichen Kreislaufstillständen, dafür aber eine Zunahme an Notrufen vor einer Wiederbelebungssituation nachweisen, was $\mathrm{zu}$ einem verbesserten initialen Überleben und einer höheren Überlebensrate bis zur Entlassung führte [210].

\section{Advanced-Life-Support(ALS)-Kurs}

Die Zielgruppe für dieses Kursformat sind Ärzte, Krankenpflegekräfte, Rettungsdienst- und spezielle innerklinische Mitarbeiter, die Leiter eines Reanimationsteams oder ein Teammitglied bei der Reanimation eines Erwachsenen werden können [211, 212].

Neben den erwarteten BLS- und ILSKompetenzen werden in diesen Kursen das Management von Kreislaufstillständen unterschiedlicher Ursachen und das Bewältigen von Peri-Arrest-Problemen geschult. Ebenso steht die Anwendung der nicht technischen Fertigkeiten zur Zusammenarbeit im Team und klare Teamführung im Unterricht im Mittelpunkt.

\section{Newborn-Life-Support(NLS)-Kurs}

Dieser interprofessionelle Eintageskurs vermittelt Mitarbeitern im Gesundheitswesen, die regelhaft mit Geburten betraut sind (beispielsweise Hebammen [213], Krankenpflege- und Rettungsdienstpersonal sowie Ärzte), das Hintergrundwissen sowie die Fertigkeiten zur Herangehensweise an das Neugeborene und dessen Wiederbelebung innerhalb der ersten 10 bis 20 Lebensminuten. Die NLS-Kurse konzentrieren sich auf Atemwegsmanagement, Thoraxkompressionen, Nabelvenenkatheter sowie Medikamentengabe bei Neugeborenen-CPR [214].

\section{European-Paediatric-Immediate-Life- Support(EPILS)-Kurs}

EPILS ist ein Eintageskurs (5 bis $8 \mathrm{~h}$ ), welcher sich an Krankenpflege- und Rettungsdienstpersonal sowie Ärzte richtet, die nicht Teil eines pädiatrischen Reanimationsteams sind. Ziel ist es, kritisch kranke Säuglinge und Kinder zu erkennen, den Kreislaufstillstand zu verhindern und Kinder im Kreislaufstillstand in den ersten Minuten bis zum Eintreffen des Reanimationsteams zu versorgen.
Kurze praktische Szenarien, angepasst an das Arbeitsumfeld und die institutionelle Stellung der Teilnehmer, werden zur Vermittlung der Kernkompetenzen genutzt.

\section{European-Paediatric-Advanced-Life- Support(EPALS)-Kurs}

EPALS richtet sich an Mitarbeiter des Gesundheitswesens, welche in die Wiederbelebung von Neugeborenen, Säuglingen und Kindern involviert sind, um innerhalb der ersten Stunden kritisch kranke oder verletzte Kinder suffizient versorgen zu können [215-218]. Eine Wiederholung der Basismaßnahmen sowie der Fremdkörperentfernung sind Bestandteile dieses Kurses.

EPALS setzt seinen Schwerpunkt auf das Erkennen sowie das regelmäßige Überprüfen und zeitgerechte Behandeln des kritisch kranken Kindes (beispielsweise durch Simulationen von Herzoder Atemversagen, Wiederbelebungssituationen sowie Traumafällen). Aspekte der Zusammenarbeit und Leitung einer Gruppe im Antizipieren von Problemen und verbessertes Situationsbewusstsein werden anhand von Schulungen vertieft. Abhängig von den lokalen Umständen und Bedürfnissen können EPALS-Kurse darüber hinaus auch Module zur Neugeborenenversorgung, zur Postreanimationsbehandlung und zu Übergabesituationen und/oder Module zu erweitertem Wissen oder technischen Fertigkeiten beinhalten. Diese zuletzt erwähnten werden kontinuierlich weiterentwickelt.

\section{Generic Instructor Course (GIC)}

Der GIC richtet sich an Teilnehmer aller ERC-Kurse (sowie angegliederter Kursformate wie dem European Trauma Course, ETC), die eine Empfehlung als „Instruktorenpotenzial“ erhalten haben. Ausgenommen sind die BLS-/AED-Kurse, für die es einen separaten Instruktorenkurs gibt. Beim GIC liegen die Schwerpunkte auf der Entwicklung der eigenen Lehrtätigkeit, dem Geben von konstruktivem sowie korrigierendem Feedback und der Mentorentätigkeit. Die Kernkompetenzen der originären Provider-Kurse werden vorausgesetzt.

Ein ERC-Educator leitet den Lernprozess und die Diskussionen und gibt kritisches Feedback. Er hält interaktive Lehr- veranstaltungen zur Theorie der Erwachsenenbildung, dem effektiven Vermitteln von Fertigkeiten und simulierten Szenarien, dem Prüfen und effektiven Feedbackgeben, wie Führungsaufgaben und Leitungsfunktionen wahrgenommen werden, und zu den nicht technischen Fertigkeiten. Die GIC-Faculty demonstriert diese Kompetenzen und schließt Übungsmöglichkeiten für die Teilnehmer an.

Gekürzte Unterrichtsmaterialien der originären Provider-Kursformate werden für die simulierten Lehrveranstaltungen genutzt. Der GIC fördert das Konzept des konstruktiven und korrigierenden Feedbacks, um zukünftige Lernstrategien zu entwickeln, welche es jedem Teilnehmer ermöglichen sollen, die Instruktorenrolle zu übernehmen.

\section{Educator Master Class (EMC)}

ERC-Educatoren sind ein essenzieller und unabdingbarer Bestandteil jeder GIC-Faculty. In diesem zweitägigen Educator-Master-Class-Kurs werden erfahrene Kursinstruktoren mit nachweislichem Interesse an der Lehre zum ERC-Educator fortgebildet. Die NRCs benennen geeignete Kandidaten, welche dann von der ERC Working Group on Education anhand spezieller vorgegebener Kriterien (inklusive Motivationsschreiben, Qualifikationen in der Medizindidaktik oder dokumentierter mehrjähriger Lehrtätigkeit im ERC) bewertet und dann als Teilnehmer der Master-Class zugelassen werden.

EMC-Instruktoren sind von der Working Group on Education und dem ERCDirektor für Training und Lehre berufene und erfahrene Educatoren. Die EMC deckt die theoretischen Hintergründe der Educatoren ab, Prüfmethodik, Qualitätskontrolle, Lehrmethodik und deren kritische Bewertung, die Mentorenrolle, die multiprofessionellen Lehrstrategien sowie die kontinuierliche Weiterentwicklung des ERC-Lehrkörpers. Das Format der EMC beinhaltet eine Reihe von geschlossenen Diskussionen, kleinen Arbeitsgruppen und Problemlösungseinheiten. Die Teilnehmer werden während des EMC formativ überprüft. 


\section{European Resuscitation \\ Academy (ERA) - „Es \\ bedarf eines Systems, um Leben zu retten"}

Die ERA hat das Ziel, das Überleben nach einem Kreislaufstillstand dadurch zu verbessern, dass sie den Fokus auf Interventionen im Gesundheitssystem legt, welches die Glieder der Überlebenskette und der Überlebensformel zusammenfügt. Das gesamte notfallmedizinische Personal von Rettungssystemen (Führungskräfte, Verwaltungs- und medizinische Leitungskräfte, Ärzte, Rettungsdienst- und Leitstellenpersonal) aus verschiedenen Gesundheitssystemen und Ländern wird eingeladen, vom ERA-Programm (welches sich von der US-amerikanischen Reanimationsakademie in Seattle (http://www.resuscitationacademy.com/) und deren „zehn Schritte für die Verbesserung des Überlebens nach Herzkreislaufstillstand" ableitet und der veranstaltenden lokalen Gesundheitseinrichtung zu lernen. Die ERA legt ihre Schwerpunkte auf die Erfassung der lokalen Überlebensrate durch das Verständnis der Bedeutung standardisierter Datenerhebung nach dem Utstein-System. Teilnehmende Rettungsdienste werden ermuntert, konkrete Maßnahmen in ihrem Umfeld zu entwickeln, die die Überlebensrate nach einem Kreislaufstillstand steigern, und diese Ereignisse entsprechend aufzuzeichnen, um den Erfolg dieser Aktionspläne zu dokumentieren.

\section{Zukünftige CPR-Lehrforschung und -Kursentwicklung}

Die Erstellung von internationalen Reanimationsleitlinien ist eine sich permanent weiterentwickelnde Aufgabe. Forschungsergebnisse von hoher Güte sollen weiterhin publiziert werden und können aufzeigen, ob die heute gültigen Leitlinien weiterhin akzeptabel sind oder nicht.

Parallel dazu entwickelt sich auch kontinuierlich die Lern- und Lehrforschung weiter. Unsere heutigen Methoden zur Vermittlung der Leitlinien unterscheiden sich erheblich von der früheren rein frontalen Vermittlung von theoretischem Wissen hin zur interaktiven und praxisnahen Lehre, die auch moderne Technologien und die sozialen Medien nutzen.
Es besteht aktuell ein Mangel an qualitativ hochwertiger Evidenz über die besten Lehrmethoden. Hintergrund dafür ist die enorme Teilnehmerzahl, die für solche Studien notwendig ist, um statistisch signifikante und bedeutungsvolle Aussagen (wie verbessertes Überleben) treffen zu können.

Es besteht daher ein Bedarf an internationaler Zusammenarbeit, um solche Testergebnisse zu erhalten, ähnlich wie durch internationale Kooperation die klinischen Inhalte der Leitlinien überprüft werden. Bis solche statistisch signifikanten Daten vorliegen, müssen die Lehrmethoden kontinuierlich evaluiert werden, um die Bedeutung oder Zielführung des gegebenen Unterrichts zu erfassen.

Neue Einblicke in den Lernprozess, den Einfluss der Neurowissenschaften auf das Training und die rasche Entwicklung der sozialen Medien sowie der internetbasierten Applikationen deuten darauf hin, dass sich unsere Herangehensweise an die Lehre permanent verändert. Dieser Abschnitt hebt aktuelle und zukünftige Änderungen hervor.

\section{Empfehlungen zur Lehrforschung in der Reanimation}

Jede Lehrintervention muss evaluiert werden, um sicherzustellen, dass alle Lernziele erreicht wurden und sich in letzter Konsequenz im besten Fall das Überleben bei Kreislaufstillstand verbessert hat. Dabei ist es nicht nur das Ziel, dass Lernende Fertigkeiten und Wissen erwerben, sondern es muss ihnen auch gelingen, diese Kompetenzen zu behalten und in adäquate Handlungen umsetzen, je nach Trainingsniveau. Eine Evaluation dieser Maßnahmen, bezogen auf Patienten-Outcome, ist schwierig zu erreichen, da sich verschiedene andere Einflüsse auswirken, wie beispielsweise Veränderungen von Leitlinien, von Patienten und ihren Erkrankungen oder von organisatorischen Faktoren. Die zu untersuchenden Ziele müssen bereits in der Planungsphase der Lehrveranstaltung festgelegt werden [219]. Verhalten in der klinischen Umgebung ist oft schwierig zu beurteilen, deshalb wird es häufig mithilfe von Simulationspuppen trainiert. Leider ist aber die Generalisierbarkeit von Studien an Simulationspuppen unzuver- lässig, was eine Ursache für die wenig verlässliche Evidenz in der Literatur ist.

Die Lehre der Reanimation ist ein relativ neues Feld mit aktuell noch fehlenden qualitativ hochwertigen Forschungsergebnissen. Die vorhandenen Studien weisen heterogene Designs auf und haben oft unerwünschte und nicht geplante Einflussfaktoren auf die Ergebnisse, weshalb diese nur schwer vergleichbar sind. Bei einem Wissenschaftsgipfel wurde nun ein Forschungskompass entwickelt, der künftige Studien in der Lehrforschung klar leiten soll [220].

\section{Zukünftige Kursentwicklung}

Die Lehrstrategie des ERC basiert auf einheitlichen, standardisierten Curricula der Instruktoren- und Anwenderkurse (Providerkurse). Dies wird sich künftig ändern, sobald mehr unterschiedliche und miteinander kombinierte Lehrmethoden verfügbar sind. Um den Lernfortschritt zu steigern, wird größere Flexibilität auf allen Ebenen der CPR-Lehre notwendig sein, vom Einsatz von DVDs bis hin zu internetbasierten bzw. Online-Kursen.

Neue Curricula sollen diese Flexibilität erlauben. Einige Module zu den Kerninhalten der Reanimation werden das „Herz“ eines jeden ERC-Kurses bilden. Daneben wird es einzelne, für die Teilnehmer maßgeschneiderte Kursformate mit zusätzlichen optionalen Inhalten (medizinische wie nicht technische Aspekte) nach den lokalen Bedürfnissen geben. Einige Institutionen werden für spezielle Teilnehmergruppen neben den Kerninhalten des Kurses auch sehr spezielle Module anbieten können (Kreislaufstillstände nach kardiochirurgischen Eingriffen, erweiterte Reanimationsmaßnahmen auf einer neonatologischen Intensivstation, geburtshilfliche Reanimationen, Reanimation während operativer Eingriffe).

Neue Unterrichtstechnologien (internetbasierte Seminare, Webinars, e-Learning-Module auf der virtuellen ERCLernplattform) werden eingeführt, die im GIC sowie in der Supervision und Metorenarbeit mit alle Instruktoren, Kursdirektoren und Educatoren erarbeitet werden müssen.

Lernende, die ein Video- oder Online-Training nutzen, werden nicht län- 
ger ein gedrucktes Manual benötigen, da sie einen einfachen Zugang zu den entsprechenden Inhalten im Internet haben. Dies wird massiv neue Möglichkeiten eröffnen, wie beispielsweise die Integration von Bildern, Demonstrationsvideos von Fertigkeiten oder der Teamarbeit, Tests zur Selbstüberprüfung mit Hinweisen, wie Mängel verbessert werden können, und für Interessierte Verlinkungen zur vertiefenden Literatur. Zudem wird es die neue virtuelle Lernumgebung des ERC den Providern, wie auch den Instruktoren und Kursorganisatoren ermöglichen, Lernfortschritte Einzelner, deren Fertigkeiten und Einstellungen sowie die Gesamtleistung auszuwerten und so weiteres Lernen der CPR zu unterstützen.

Bevor Teilnehmer den Kursort erreichen, können so wissensbasierte Fakten gelesen und gelernt, Prozeduren und Handlungsstrategien durchgedacht und offene Fragen diskutiert werden. Dadurch kommen hochmotivierte Teilnehmer in die Kurszentren zum Präsenzunterricht. Sie sind vertraut mit den Inhalten und haben klare Vorstellungen von Interaktionen im Team und davon, welche Maßnahmen für hochwertige CPR notwendig sind. Da aus wirtschaftlichen Gründen meistens keine Zeit für Weiterbildungen ist, werden die Kurse auf die Übertragung der erlernten Inhalte in den Simulationsszenarien fokussieren. Dies ermöglicht den Teilnehmern das Ausprobieren, Einüben und Ausführen der lebensrettenden Techniken vor dem Hintergrund bestmöglicher medizinischer Praxis, entsprechender Leitungskompetenz und Management im Team. Schließlich soll dies den Anwendern im klinischen Einsatz ermöglichen, das Überleben nach einem Kreislaufstillstand zu verbessern.

Häufige stattfindende CPR-Trainingskurse werden nur noch sehr kurz dauern und nicht notwendigerweise durch einen Instruktor oder Mentor geleitet werden müssen. Die Lernumgebung muss zum Lernenden gebracht werden, sodass dieser in seiner täglichen Praxis üben und somit den gewünschten Lernerfolg vor Ort erfahren kann. Ein kurzer jährlicher CPRKompetenztest kann diejenigen herausfiltern, die nicht das institutionell festgelegte Kompetenzniveau erreichen. Einige werden ein kurzes supervisiertes Training be- nötigen, während andere einen längeren Wiederauffrischungskurs besuchen müssen. Die Organisatoren müssen ihre Kurse daher flexibel planen, um bestimmten Zielgruppen mit besonderen Hintergrundkompetenzen eine möglichst kurze CPR-Kursanwesenheit zu ermöglichen und Laienhelfern mehr Zeit für eine praxisorientierte Ausbildung zu geben.

Der Einsatz von High-Fidelity-Simulationspuppen und fortgeschrittenen Feedback-Geräten wird nur in finanzkräftigen Ländern und Organisationen verfügbar sein. Werden einfachere wie die herkömmlichen CPR-Puppen benutzt, müssen Instruktoren so geschult werden, dass sie zeitgerechtes und valides Feedback an die Lernenden geben, um deren Lernerfolg zu steigern.

Zusammenfassend ist es das Ziel des ERC, die Glieder der Überlebenskette durch effektive Lehre und Implementierung von hochwertiger CPR zu stärken. Dies setzt voraus, dass Lernstrategien sowohl für Laienhelfer als auch für Profis im Gesundheitswesen entwickelt werden, mit denen qualitativ hochwertige BLS, zügige Defibrillation, effektive erweiterte Reanimationsmaßnahmen sowie qualitativ hochwertige Postreanimationsbehandlung zu erreichen ist. Diese Strategien müssen einfach, für jedermann zugänglich, gut validiert sowie ansprechend sein. So wird sichergestellt, dass die wissenschaftlichen Leitlinien effektiv in verbesserte Überlebensraten umgesetzt werden können.

\section{Korrespondenzadresse}

\section{R. Greif}

Department of Anaesthesiology and Pain Medicine

University Hospital Bern, University of Bern

Bern

robert.greif@insel.ch

\section{Korrespondierender Übersetzer}

\section{Robert Greif}

Universitätsklinik für Anästhesiologie und Schmerztherapie,

Universitätsspital Bern, Inselspital, und Universität Bern,

Bern, Schweiz

\section{Thomas Ahne}

Freiburg

\section{Einhaltung ethischer Richtlinien}

Interessenkonflikt. R. Greif ist Editor in Trends in Anesthesia and Critical Care; A. Lockey ist medizinischer Berater von "First on Scene First Aid company"; W. De Vries ist Angestellter der Training Organisation ACM; A. Lippert, K. Monsieurs und P. Conoghan geben an, dass kein Interessenkonflikt besteht.

\section{Literatur}

1. Nolan J, Soar J, Eikeland H (2006) The chain of survival. Resuscitation 71:270-271

2. Soreide E, Morrison L, Hillman K et al (2013) The formula for survival in resuscitation. Resuscitation 84:1487-1493

3. Chamberlain DA, Hazinski MF (2003) Education in resuscitation. Resuscitation 59:11-43

4. Morley PT, Lang E, Aickin R et al (2015) Part 2: evidence evaluation and management of conflict of interest for the ILCOR 2015 consensus on science and treatment recommendations. Resuscitation (im Druck)

5. Berdowski J, Schmohl A, Tijssen JG, Koster RW (2009) Time needed for a regional emergency medical system to implement resuscitation Guidelines 2005 - The Netherlands experience. Resuscitation 80:1336-1341

6. Bigham BL, Aufderheide TP, Davis DP et al (2010) Knowledge translation in emergency medical services: a qualitative survey of barriers to guideline implementation. Resuscitation 81:836-840

7. Bigham BL, Koprowicz K, Aufderheide TP et al (2010) Delayed prehospital implementation of the 2005 American Heart Association guidelines for cardiopulmonary resuscitation and emergency cardiac care. Prehosp Emerg Care 14:355-360

8. Kudenchuk PJ, Redshaw JD, Stubbs BA et al (2012) Impact of changes in resuscitation practice on survival and neurological outcome after out-of-hospital cardiac arrest resulting from nonshockable arrhythmias. Circulation 125:1787-1794

9. Steinberg MT, Olsen JA, Brunborg C et al (2015) Minimizing pre-shock chest compression pauses in a cardiopulmonary resuscitation cycle by performing an earlier rhythm analysis. Resuscitation 87:33-37

10. Swor R, Khan I, Domeier R, Honeycutt L, Chu K, Compton S (2006) CPR training and CPR performance: do CPR-trained bystanders perform CPR? Acad Emerg Med 13:596-601

11. Tanigawa K, Iwami T, Nishiyama C, Nonogi H, Kawamura T (2011) Are trained individuals more likely to perform bystander CPR? An observational study. Resuscitation 82:523-528

12. Nielsen AM, Isbye DL, Lippert FK, Rasmussen LS (2013) Can mass education and a television campaign change the attitudes towards cardiopulmonary resuscitation in a rural community? Scand J Trauma Resusc Emerg Med 21:39

13. Savastano S, Vanni V (2011) Cardiopulmonary resuscitation in real life: the most frequent fears of lay rescuers. Resuscitation 82:568-571

14. Sasson C, Haukoos JS, Bond C et al (2013) Barriers and facilitators to learning and performing cardiopulmonary resuscitation in neighborhoods with low bystander cardiopulmonary resuscitation prevalence and high rates of cardiac arrest in Columbus, $\mathrm{OH}$. Circ Cardiovasc Qual Outcomes 6:550-558 
15. King R, Heisler M, Sayre MR et al (2015) Identification of factors integral to designing communitybased CPR interventions for high-risk neighborhood residents. Prehosp Emerg Care 19:308-312

16. Greenberg MR, Barr GC Jr, Rupp VA et al (2012) Cardiopulmonary resuscitation prescription program: a pilot randomized comparator trial. J Emerg Med 43:166-171

17. Blewer AL, Leary M, Esposito EC et al (2012) Continuous chest compression cardiopulmonary resuscitation training promotes rescuer self-confidence and increased secondary training: a hospital-based randomized controlled trial* . Crit Care Med 40:787-792

18. Brannon TS, White LA, Kilcrease JN, Richard LD, Spillers JG, Phelps CL (2009) Use of instructional video to prepare parents for learning infant cardiopulmonary resuscitation. Proc (Bayl Univ Med Cent) 22:133-137

19. Haugk M, Robak O, Sterz F et al (2006) High acceptance of a home AED programme by survivors of sudden cardiac arrest and their families. Resuscitation 70:263-274

20. Knight LJ, Wintch S, Nichols A, Arnolde V, Schroeder AR (2013) Saving a life after discharge: CPR training for parents of high-risk children. J Healthc Qual 35:9-16 (quiz7)

21. Barr GC Jr, Rupp VA, Hamilton KM et al (2013) Training mothers in infant cardiopulmonary resuscitation with an instructional DVD and manikin. J Am Osteopath Assoc 113:538-545

22. Plant N, Taylor K (2013) How best to teach CPR to schoolchildren: a systematic review. Resuscitation $84: 415-421$

23. Cave DM, Aufderheide TP, Beeson J et al (2011) Importance and implementation of training in cardiopulmonary resuscitation and automated external defibrillation in schools: a science advisory from the American Heart Association. Circulation 123:691-706

24. Wissenberg M, Lippert FK, Folke F et al (2013) Association of national initiatives to improve cardiac arrest management with rates of bystander intervention and patient survival after out-ofhospital cardiac arrest. Jama 310:1377-1384

25. Bohn A, Van Aken HK, MollhoffT et al (2012) Teaching resuscitation in schools: annual tuition by trained teachers is effective starting at age 10. A four-year prospective cohort study. Resuscitation 83:619-625

26. Stroobants J, Monsieurs K, Devriendt B, Dreezen C, Vets P, Mols P (2014) Schoolchildren as BLS instructors for relatives and friends: impact on attitude towards bystander CPR. Resuscitation 85:1769-1774

27. Stiell IG, Brown SP, Christenson J et al (2012) What is the role of chest compression depth during out-of-hospital cardiac arrest resuscitation?*. Crit Care Med 40:1192-1198

28. Song KJ, Shin SD, Park CB et al (2014) Dispatcherassisted bystander cardiopulmonary resuscitation in a metropolitan city: a before - after population-based study. Resuscitation 85:34-41

29. Lewis M, Stubbs BA, Eisenberg MS (2013) Dispatcher-assisted cardiopulmonary resuscitation: time to identify cardiac arrest and deliver chest compression instructions. Circulation 128:15221530

30. Bohm K, Stalhandske B, Rosenqvist M, Ulfvarson J, Hollenberg J, Svensson L (2009) Tuition of emergency medical dispatchers in the recognition of agonal respiration increases the use of telephone assisted CPR. Resuscitation 80:1025-1028
31. Mancini ME, Cazzell M, Kardong-Edgren S, Cason $\mathrm{CL}$ (2009) Improving workplace safety training using a self-directed CPR-AED learning program. AAOHN J 57:159-167 (quiz 68-69)

32. Cason CL, Kardong-Edgren S, Cazzell M, Behan D, Mancini ME (2009) Innovations in basic life support education for healthcare providers: improving competence in cardiopulmonary resuscitation through self-directed learning. J Nurses Staff Dev 25:E1-E13

33. Einspruch EL, Lynch B, Aufderheide TP, Nichol G, Becker $L$ (2007) Retention of CPR skills learned in a traditional AHA Heartsaver course versus 30 min video self-training: a controlled randomized study. Resuscitation 74:476-486

34. Lynch B, Einspruch EL, Nichol G, Becker LB, Aufderheide TP, Idris A (2005) Effectiveness of a 30min CPR self-instruction program for lay responders: a controlled randomized study. Resuscitation 67:31-43

35. Chung CH, Siu AY, Po LL, Lam CY, Wong PC (2010) Comparing the effectiveness of video self-instruction versus traditional classroom instruction targeted at cardiopulmonary resuscitation skills for laypersons: a prospective randomised controlled trial. Hong Kong Med J 16:165-170

36. Andersen PO, Jensen MK, Lippert A, Ostergaard D (2010) Identifying non-technical skills and barriers for improvement of teamwork in cardiac arrest teams. Resuscitation 81:695-702

37. Flin R, Patey R, Glavin R, Maran N (2010) Anaesthetists' non-technical skills. Br J Anaesth 105:3844

38. Iwami T, Kitamura T, Kawamura T et al (2012) Chest compression-only cardiopulmonary resuscitation for out-of-hospital cardiac arrest with public-access defibrillation: a nationwide cohort study. Circulation 126:2844-2851

39. Nielsen AM, Folke F, Lippert FK, Rasmussen LS (2013) Use and benefits of public access defibrillation in a nation-wide network. Resuscitation 84:430-434

40. Harrison-Paul R, Timmons $S$, van Schalkwyk WD (2006) Training lay-people to use automatic external defibrillators: are all of their needs being met? Resuscitation 71:80-88

41. Perkins GD, Travers AH, Considine J et al (2015) Part 3: adult basic life support and automated external defibrillation: 2015 International Consensus on Cardiopulmonary Resuscitation and Emergency Cardiovascular Care Science With Treatment Recommendations. Resuscitation (im Druck)

42. Perkins GD, Handley AJ, Koster KW et al (2015) European Resuscitation Council Guidelines for Resuscitation 2015 Section 2 Adult basic life support and automated external defibrillation. Resuscitation. http://dx.doi.org/10.1016/j.resuscitation.2015.07.015

43. Yeung J, Meeks R, Edelson D, Gao F, Soar J, Perkins GD (2009) The use of CPR feedback/prompt devices during training and CPR performance: $a$ systematic review. Resuscitation 80:743-51

44. Maconochie I, Bingham R, Eich C et al (2015) European Resuscitation Council Guidelines for Resuscitation 2015 Section 6 Paediatric Life Support. Resuscitation. http://dx.doi.org/10.1016/j. resuscitation.2015.07.028

45. Hoke RS, Chamberlain DA, Handley AJ (2006) A reference automated external defibrillator provider course for Europe. Resuscitation 69:421-433
46. Roppolo LP, Pepe PE, Campbell L et al (2007) Prospective, randomized trial of the effectiveness and retention of 30-min layperson training for cardiopulmonary resuscitation and automated external defibrillators: The American Airlines Study. Resuscitation 74:276-285

47. Isbye DL, Rasmussen LS, Lippert FK, Rudolph SF, Ringsted CV (2006) Laypersons may learn basic life support in 24 min using a personal resuscitation manikin. Resuscitation 69:435-442

48. de Vries W, Turner NM, Monsieurs KG, Bierens JJ, Koster RW (2010) Comparison of instructor-led automated external defibrillation training and three alternative DVD-based training methods. Resuscitation 81:1004-1009

49. Reder S, Cummings P, Quan L (2006) Comparison of three instructional methods for teaching cardiopulmonary resuscitation and use of an automatic external defibrillator to high school students. Resuscitation 69:443-453

50. Roppolo LP, Heymann R, Pepe P et al (2011) A randomized controlled trial comparing traditional training in cardiopulmonary resuscitation (CPR) to self-directed CPR learning in first year medical students: the two-person CPR study. Resuscitation 82:319-325

51. Yeung J, Okamoto D, Soar J, Perkins GD (2011) AED training and its impact on skill acquisition, retention and performance-a systematic review of alternative training methods. Resuscitation 82:657-664

52. Deakin CD, Shewry E, Gray HH (2014) Public access defibrillation remains out of reach for most victims of out-of-hospital sudden cardiac arrest. Heart 100:619-623

53. Smith KK, Gilcreast D, Pierce K (2008) Evaluation of staff's retention of ACLS and BLS skills. Resuscitation 78:59-65

54. Woollard M, Whitfeild R, Smith A et al (2004) Skill acquisition and retention in automated external defibrillator (AED) use and CPR by lay responders: a prospective study. Resuscitation 60:17-28

55. Woollard M, Whitfield R, Newcombe RG, Colquhoun M, Vetter N, Chamberlain D (2006) Optimal refresher training intervals for $A E D$ and $C P R$ skills: a randomised controlled trial. Resuscitation 71:237-247

56. Andresen D, Arntz HR, Grafling W et al (2008) Public access resuscitation program including defibrillator training for laypersons: a randomized trial to evaluate the impact of training course duration. Resuscitation 76:419-424

57. Beckers SK, Fries M, Bickenbach J et al (2007) Retention of skills in medical students following minimal theoretical instructions on semi and fully automated external defibrillators. Resuscitation 72:444-450

58. de Vries W, Handley AJ (2007) A web-based micro-simulation program for self-learning BLS skills and the use of an AED. Can laypeople train themselves without a manikin? Resuscitation 75:491498

59. Jerin JM, Ansell BA, Larsen MP, Cummins RO (1998) Automated external defibrillators: skill maintenance using computer-assisted learning. Acad Emerg Med 5:709-717

60. Bobrow BJ, Vadeboncoeur TF, Spaite DW et al (2011) The effectiveness of ultrabrief and brief educational videos for training lay responders in hands-only cardiopulmonary resuscitation: implications for the future of citizen cardiopulmonary resuscitation training. Circ Cardiovasc Qual Outcomes 4:220-226 
61. Sutton RM, Niles D, Meaney PA et al (2011) „Booster" training: evaluation of instructor-led bedside cardiopulmonary resuscitation skill training and automated corrective feedback to improve cardiopulmonary resuscitation compliance of Pediatric Basic Life Support providers during simulated cardiac arrest. Pediatr Crit Care Med 12:e116e121

62. Sutton RM, Niles D, Meaney PA et al (2011) Lowdose, high-frequency CPR training improves skill retention of in-hospital pediatric providers. Pediatrics 128:e145-e151

63. Harvey PR, Higenbottam CV, Owen A, Hulme J, Bion JF (2012) Peer-led training and assessment in basic life support for healthcare students: synthesis of literature review and fifteen years practical experience. Resuscitation 83:894-899

64. Spooner BB, Fallaha JF, Kocierz L, Smith CM, Smith SC, Perkins GD (2007) An evaluation of objective feedback in basic life support (BLS) training. Resuscitation 73:417-424

65. Kitamura T, Iwami T, Kawamura T et al (2010) Conventional and chest-compression-only cardiopulmonary resuscitation by bystanders for children who have out-of-hospital cardiac arrests: a prospective, nationwide, population-based cohort study. Lancet 375:1347-1354

66. Castle N, Garton H, Kenward G (2007) Confidence vs competence: basic life support skills of health professionals. Br J Nurs 16:664-666

67. Wik L, Myklebust $H$, Auestad BH, Steen PA (2005) Twelve-month retention of CPR skills with automatic correcting verbal feedback. Resuscitation 66:27-30

68. Christenson J, Nafziger S, Compton S et al (2007) The effect of time on CPR and automated external defibrillator skills in the Public Access Defibrillation Trial. Resuscitation 74:52-62

69. Niles D, Sutton RM, Donoghue A et al (2009) „Rolling Refreshers": a novel approach to maintain CPR psychomotor skill competence. Resuscitation 80:909-912

70. Beckers SK, Skorning MH, Fries M et al (2007) CPREzy improves performance of external chest compressions in simulated cardiac arrest. Resuscitation 72:100-107

71. Nishisaki A, Nysaether J, Sutton R et al (2009) Effect of mattress deflection on CPR quality assessment for older children and adolescents. Resuscitation 80:540-545

72. Perkins GD, Kocierz L, Smith SC, McCulloch RA, Davies RP (2009) Compression feedback devices over estimate chest compression depth when performed on a bed. Resuscitation 80:79-82

73. Kirkbright S, Finn J, Tohira H, Bremner A, Jacobs I, Celenza A (2014) Audiovisual feedback device use by health care professionals during CPR: a systematic review and meta-analysis of randomised and non-randomised trials. Resuscitation 85:460-471

74. Yeung J, Davies R, Gao F, Perkins GD (2014) A randomised control trial of prompt and feedback devices and their impact on quality of chest compressions-a simulation study. Resuscitation 85:553-559

75. Zapletal B, Greif R, Stumpf D et al (2014) Comparing three CPR feedback devices and standard BLS in a single rescuer scenario: a randomised simulation study. Resuscitation 85:560-566

76. Cheng A, Brown LL, Duff JP et al (2015) Improving cardiopulmonary resuscitation with a CPR feedback device and refresher simulations (CPR CARES Study): a randomized clinical trial. JAMA pediatr 169:137-144
77. Clark LJ, Watson J, Cobbe SM, Reeve W, Swann IJ, Macfarlane PW (2000) CPR'98: a practical multimedia computer-based guide to cardiopulmonary resuscitation for medical students. Resuscitation 44:109-117

78. Hudson JN (2004) Computer-aided learning in the real world of medical education: does the quality of interaction with the computer affect student learning? Medical Educ 38:887-895

79. Jang KS, Hwang SY, Park SJ, Kim YM, Kim MJ (2005) Effects of a Web-based teaching method on undergraduate nursing students' learning of electrocardiography. J Nurs Educ 44:35-39

80. Leong SL, Baldwin CD, Adelman AM (2003) Integrating Web-based computer cases into a required clerkship: development and evaluation. Acad Med 78:295-301

81. Rosser JC, Herman B, Risucci DA, Murayama M, Rosser LE, Merrell RC (2000) Effectiveness of a CD-ROM multimedia tutorial in transferring cognitive knowledge essential for laparoscopic skill training. Am J Surg 179:320-324

82. Papadimitriou L, Xanthos T, Bassiakou E, Stroumpoulis K, Barouxis D, lacovidou N (2010) Distribution of pre-course BLS/AED manuals does not influence skill acquisition and retention in lay rescuers: a randomised study. Resuscitation 81:348-352

83. Perkins GD, Fullerton JN, Davis-Gomez $\mathrm{N}$ et al (2010) The effect of pre-course e-learning prior to advanced life support training: a randomised controlled trial. Resuscitation 81:877-881

84. Perkins GD, Kimani PK, Bullock I et al (2012) Improving the efficiency of advanced life support training: a randomized, controlled trial. Ann Intern Med 157:19-28

85. Thorne CJ, Lockey AS, Bullock I et al (2015) E-learning in advanced life support-an evaluation by the Resuscitation Council (UK). Resuscitation 90:79-84

86. Orde S, Celenza A, Pinder M (2010) A randomised trial comparing a 4-stage to 2-stage teaching technique for laryngeal mask insertion. Resuscitation 81:1687-1691

87. Greif R, Egger L, Basciani RM, Lockey A, Vogt $A$ (2010) Emergency skill training-a randomized controlled study on the effectiveness of the 4-stage approach compared to traditional clinical teaching. Resuscitation 81:1692-1697

88. Mundell WC, Kennedy CC, Szostek JH, Cook DA (2013) Simulation technology for resuscitation training: a systematic review and meta-analysis. Resuscitation 84:1174-1183

89. Cheng A, Lang TR, Starr SR, Pusic M, Cook DA (2014) Technology-enhanced simulation and pediatric education: a meta-analysis. Pediatrics 133:e1313-e1323

90. Cheng A, Lockey A, Bhanji F, Lin Y, Hunt EA, Lang $E$ (2015) The use of high-fidelity manikins for advanced life support training-A systematic review and meta-analysis. Resuscitation 93:142-149

91. Krogh KB, Hoyer CB, Ostergaard D, Eika B (2014) Time matters-realism in resuscitation training. Resuscitation 85:1093-1098

92. Hunt EA, Cruz-Eng H, Bradshaw JH et al (2015) A novel approach to life support training using ${ }_{\text {IIC- }}$ tion-linked phrases" Resuscitation 86:1-5

93. Hunt EA, Duval-Arnould JM, Nelson-McMillan KL et al (2014) Pediatric resident resuscitation skills improve after "rapid cycle deliberate practice" training. Resuscitation 85:945-951
94. Hunziker S, Buhlmann C, Tschan F et al (2010) Brief leadership instructions improve cardiopulmonary resuscitation in a high-fidelity simulation: a randomized controlled trial. Crit Care Med 38:1086-1091

95. Hunziker S, Tschan F, Semmer NK et al (2009) Hands-on time during cardiopulmonary resuscitation is affected by the process of teambuilding: a prospective randomised simulator-based trial. BMC Emerg Med 9:3

96. Andreatta P, Saxton E, Thompson M, Annich G (2011) Simulation-based mock codes significantly correlate with improved pediatric patient cardiopulmonary arrest survival rates. Pediatr Crit Care Med 12:33-38

97. Neily J, Mills PD, Young-Xu Y et al (2010) Association between implementation of a medical team training program and surgical mortality. JAMA 304:1693-1700

98. Boet S, Bould MD, Fung L et al (2014) Transfer of learning and patient outcome in simulated crisis resource management: a systematic review. Can J Anaesth 61:571-582

99. Rall M, Gaba DM, Dieckmann RA (2010) Patient Simulation. In: Miller RD (Hrsg) Anesthesia. Elsevier, New York, S 151-192

100. Thomas EJ, Taggart B, Crandell S et al (2007) Teaching teamwork during the Neonatal Resuscitation Program: a randomized trial. J Perinatol 27:409-414

101. Gilfoyle E, Gottesman R, Razack S (2007) Development of a leadership skills workshop in paediatric advanced resuscitation. Med Teach 29:e276-e283

102. Edelson DP, Litzinger $B$, Arora V et al (2008) Improving in-hospital cardiac arrest process and outcomes with performance debriefing. Arch Inter Med 168:1063-1069

103. Hayes CW, Rhee A, Detsky ME, Leblanc VR, Wax RS (2007) Residents feel unprepared and unsupervised as leaders of cardiac arrest teams in teaching hospitals: a survey of internal medicine residents. Crit Care Med 35:1668-1672

104. Marsch SC, Muller C, Marquardt K, Conrad G, Tschan F, Hunziker PR (2004) Human factors affect the quality of cardiopulmonary resuscitation in simulated cardiac arrests. Resuscitation 60:5156

105. Salas E, DiazGranados D, Weaver SJ, King H (2008) Does team training work? Principles for health care. Acad Emerg Med 15:1002-1009

106. Eppich W, Howard V, Vozenilek J, Curran I (2011) Simulation-based team training in healthcare. Simul Healthc 6(Suppl):S14-S19

107. Thomas EJ, Williams AL, Reichman EF, Lasky RE, Crandell S, Taggart WR (2010) Team training in the neonatal resuscitation program for interns: teamwork and quality of resuscitations. Pediatrics 125:539-546

108. Garbee DD, Paige J, Barrier K et al (2013) Interprofessional teamwork among students in simulated codes: a quasi-experimental study. Nurs Educ perspect 34:339-344

109. Chung SP, Cho J, Park YS et al (2011) Effects of script-based role play in cardiopulmonary resuscitation team training. Emerg Med J 28:690-694

110. Yeung JH, Ong GJ, Davies RP, Gao F, Perkins GD (2012) Factors affecting team leadership skills and their relationship with quality of cardiopulmonary resuscitation. Crit Care Med 40:26172621 
111. Blackwood J, Duff JP, Nettel-Aguirre A, Djogovic D, Joynt C (2014) Does teaching crisis resource management skills improve resuscitation performance in pediatric residents?*. Pediatr Crit Care Med 15:e168-e174

112. Weidman EK, Bell G, Walsh D, Small S, Edelson DP (2010) Assessing the impact of immersive simulation on clinical performance during actual inhospital cardiac arrest with CPR-sensing technology: a randomized feasibility study. Resuscitation 81:1556-1561

113. Cooper S, Cant R, Porter J et al (2010) Rating medical emergency teamwork performance: development of the Team Emergency Assessment Measure (TEAM). Resuscitation 81:446-452

114. Kim J, Neilipovitz D, Cardinal P, Chiu M (2009) A comparison of global rating scale and checklist scores in the validation of an evaluation tool to assess performance in the resuscitation of critically ill patients during simulated emergencies (abbreviated as "CRM simulator study IB"). Simul Healthc 4:6-16

115. Malec JF, Torsher LC, Dunn WF et al (2007) The mayo high performance teamwork scale: reliability and validity for evaluating key crew resource management skills. Simul Healthc 2:4-10

116. Rosen MA, Salas E, Silvestri S, Wu TS, Lazzara EH (2008) A measurement tool for simulation-based training in emergency medicine: the simulation module for assessment of resident targeted event responses (SMARTER) approach. Simul Healthc 3:170-179

117. Fischer H, Strunk G, Neuhold S et al (2012) The effectiveness of ERC advanced life support (ALS) provider courses for the retention of ALS knowledge. Resuscitation 83:227-231

118. Jensen ML, Lippert F, Hesselfeldt R et al (2009) The significance of clinical experience on learning outcome from resuscitation training-a randomised controlled study. Resuscitation 80:238243

119. Fischer H, Bachmann K, Strunk G et al (2014) Translation of ERC resuscitation quidelines into clinical practice by emergency physicians. Scand J Trauma Resusc Emerg Med 22:9

120. Rodgers DL, Bhanji F, McKee BR (2010) Written evaluation is not a predictor for skills performance in an Advanced Cardiovascular Life Support course. Resuscitation 81:453-456

121. Napier F, Davies RP, Baldock C et al (2009) Validation for a scoring system of the ALS cardiac arrest simulation test (CASTest). Resuscitation 80:10341038

122. Kromann CB, Jensen ML, Ringsted C (2009) The effect of testing on skills learning. Med Educ 43:21-27

123. Kromann $C B$, Bohnstedt $C$, Jensen $M L$, Ringsted $C$ (2010) The testing effect on skills learning might last 6 months. Adv Health Sci Educ Theory Pract 15:395-401

124. Kurosawa $H$, lkeyama T, Achuff $P$ et al (2014) A randomized, controlled trial of in situ pediatric advanced life support recertification („,pediatric advanced life support reconstructed") compared with standard pediatric advanced life support recertification for ICU frontline providers*. Crit Care Med 42:610-618

125. Patocka C, Khan F, Dubrovsky AS, Brody D, Bank I, Bhanji F (2015) Pediatric resuscitation traininginstruction all at once or spaced over time? Resuscitation $88: 6-11$

126. Stross JK (1983) Maintaining competency in advanced cardiac life support skills. JAMA 249:3339-3341
127. Jensen ML, Mondrup F, Lippert F, Ringsted C (2009) Using e-learning for maintenance of ALS competence. Resuscitation 80:903-908

128. Kaczorowski J, Levitt C, Hammond M et al (1998) Retention of neonatal resuscitation skills and knowledge: a randomized controlled trial. Fam Med 30:705-711

129. Bender J, Kennally K, Shields R, Overly F (2014) Does simulation booster impact retention of resuscitation procedural skills and teamwork? J Perinatol 34:664-668

130. Nelson KL, Shilkofski NA, Haggerty JA, Saliski M, Hunt EA (2008) The use of cognitive AIDS during simulated pediatric cardiopulmonary arrests. Simul Healthc 3:138-145

131. Mills PD, DeRosier JM, Neily J, McKnight SD, Weeks WB, Bagian JP (2004) A cognitive aid for cardiac arrest: you can't use it if you don't know about it. Jt Comm J Qual Saf 30:488-496

132. Kelleher DC, Carter EA, Waterhouse LJ, Parsons SE, Fritzeen JL, Burd RS (2014) Effect of a checklist on advanced trauma life support task performance during pediatric trauma resuscitation. Acad Emerg Med 21:1129-1134

133. Mpotos N, Lemoyne S, Calle PA, Deschepper E, Valcke M, Monsieurs KG (2011) Combining video instruction followed by voice feedback in a selflearning station for acquisition of Basic Life Support skills: a randomised non-inferiority trial. $\mathrm{Re}$ suscitation 82:896-901

134. Mpotos N, Yde L, Calle P et al (2013) Retraining basic life support skills using video, voice feedback or both: a randomised controlled trial. Resuscitation 84:72-77

135. Skorning M, Derwall M, Brokmann JC et al (2011) External chest compressions using a mechanical feedback device: cross-over simulation study. Anaesthesist 60:717-722

136. Handley AJ, Handley SA (2003) Improving CPR performance using an audible feedback system suitable for incorporation into an automated external defibrillator. Resuscitation 57:57-62

137. Woollard M, Poposki J, McWhinnie B, Rawlins L, Munro G, O'Meara P (2012) Achy breaky makey wakey heart? A randomised crossover trial of musical prompts. Emerg Med J 29:290-294

138. Oh JH, Lee SJ, Kim SE, Lee KJ, Choe JW, Kim CW (2008) Effects of audio tone guidance on performance of CPR in simulated cardiac arrest with an advanced airway. Resuscitation 79:273-277

139. Rawlins L, Woollard M, Williams J, Hallam P (2009) Effect of listening to Nellie the Elephant during CPR training on performance of chest compressions by lay people: randomised crossover trial. Bmj 339:b4707

140. Couper K, Smyth M, Perkins GD (2015) Mechanical devices for chest compression: to use or not to use? Curr Opin Crit Care 21:188-194

141. Allan CK, Thiagarajan RR, Beke D et al (2010) Simulation-based training delivered directly to the pediatric cardiac intensive care unit engenders preparedness, comfort, and decreased anxiety among multidisciplinary resuscitation teams. J Thorac Cardiovasc Surg 140:646-652

142. Lighthall GK, Poon T, Harrison TK (2010) Using in situ simulation to improve in-hospital cardiopulmonary resuscitation. Jt Comm J Qual Patient Saf 36:209-216

143. Mikrogianakis A, Osmond MH, Nuth JE, Shephard A, Gaboury I, Jabbour M (2008) Evaluation of a multidisciplinary pediatric mock trauma code educational initiative: a pilot study. J Trauma 64:761-767
144. Farah R, Stiner E, Zohar Z, Zveibil F, Eisenman A (2007) Cardiopulmonary resuscitation surprise drills for assessing, improving and maintaining cardiopulmonary resuscitation skills of hospital personnel. Eur J Emerg Med 14:332-336

145. Villamaria FJ, Pliego JF, Wehbe-Janek $\mathrm{H}$ et al (2008) Using simulation to orient code blue teams to a new hospital facility. Simul Healthc 3:209-216

146. Hunt EA, Hohenhaus SM, Luo X, Frush KS (2006) Simulation of pediatric trauma stabilization in 35 North Carolina emergency departments: identification of targets for performance improvement. Pediatrics 117:641-648

147. Hunt EA, Walker AR, Shaffner DH, Miller MR, Pronovost PJ (2008) Simulation of in-hospital pediatric medical emergencies and cardiopulmonary arrests: highlighting the importance of the first 5 min. Pediatrics 121:e34-e43

148. Raemer D, Anderson M, Cheng A, Fanning R, Nadkarni V, Savoldelli G (2011) Research regarding debriefing as part of the learning process. Simul Healthc 6(Suppl):S52-S57

149. Byrne AJ, Sellen AJ, Jones JG et al (2002) Effect of videotape feedback on anaesthetists' performance while managing simulated anaesthetic crises: a multicentre study. Anaesthesia 57:176179

150. Savoldelli GL, Naik VN, Park J, Joo HS, Chow R, Hamstra SJ (2006) Value of debriefing during simulated crisis management: oral versus videoassisted oral feedback. Anesthesiology 105:279285

151. Olasveengen TM, Vik E, Kuzovlev A, Sunde K (2009) Effect of implementation of new resuscitation guidelines on quality of cardiopulmonary resuscitation and survival. Resuscitation 80:407411

152. Aufderheide TP, Yannopoulos D, Lick CJ et al (2010) Implementing the 2005 American Heart Association Guidelines improves outcomes after out-of-hospital cardiac arrest. Heart Rhythm 7:1357-1362

153. Rea TD, Helbock M, Perry S et al (2006) Increasing use of cardiopulmonary resuscitation during outof-hospital ventricular fibrillation arrest: survival implications of guideline changes. Circulation 114:2760-2765

154. Garza AG, Gratton MC, Salomone JA, Lindholm D, McElroy J, Archer R (2009) Improved patient survival using a modified resuscitation protocol for out-of-hospital cardiac arrest. Circulation 119:2597-2605

155. Deasy C, Bray JE, Smith K et al (2011) Cardiac arrest outcomes before and after the 2005 resuscitation guidelines implementation: evidence of improvement? Resuscitation 82:984-988

156. Bigham BL, Koprowicz K, Rea T et al (2011) Cardiac arrest survival did not increase in the Resuscitation Outcomes Consortium after implementation of the 2005 AHA CPR and ECC guidelines. Resuscitation 82:979-983

157. Lund-Kordahl I, Olasveengen TM, Lorem T, Samdal M, Wik L, Sunde K (2010) Improving outcome after out-of-hospital cardiac arrest by strengthening weak links of the local Chain of Survival; quality of advanced life support and post-resuscitation care. Resuscitation 81:422-426 
158. Engdahl J, Abrahamsson P, Bang A, Lindqvist J, Karlsson T, Herlitz J (2000) Is hospital care of major importance for outcome after out-of-hospital cardiac arrest? Experience acquired from patients with out-of-hospital cardiac arrest resuscitated by the same Emergency Medical Service and admitted to one of two hospitals over a 16-year period in the municipality of Goteborg. Resuscitation 43:201-211

159. Callaway CW, Schmicker R, Kampmeyer M et al (2010) Receiving hospital characteristics associated with survival after out-of-hospital cardiac arrest. Resuscitation 81:524-529

160. Carr BG, Goyal M, Band RA et al (2009) A national analysis of the relationship between hospital factors and post-cardiac arrest mortality. Intensive Care Med 35:505-511

161. Carr BG, Kahn JM, Merchant RM, Kramer AA, Neumar RW (2009) Inter-hospital variability in postcardiac arrest mortality. Resuscitation 80:30-34

162. Davis DP, Fisher R, Aguilar S et al (2007) The feasibility of a regional cardiac arrest receiving system. Resuscitation 74:44-51

163. Fothergill RT, Watson LR, Virdi GK, Moore FP, Whitbread M (2014) Survival of resuscitated cardiac arrest patients with ST-elevation myocardial infarction (STEMI) conveyed directly to a Heart Attack Centre by ambulance clinicians. Resuscitation 85:96-98

164. Stub D, Smith K, Bray JE, Bernard S, Duffy SJ, Kaye DM (2011) Hospital characteristics are associated with patient outcomes following out-of-hospital cardiac arrest. Heart 97:1489-1494

165. Bosson N, Kaji AH, Niemann JT et al (2014) Survival and neurologic outcome after out-of-hospital cardiac arrest: results one year after regionalization of post-cardiac arrest care in a large metropolitan area. Prehosp Emerg Care 18:217-223

166. Callaway CW, Schmicker RH, Brown SP et al (2014) Early coronary angiography and induced hypothermia are associated with survival and functional recovery after out-of-hospital cardiac arrest. Resuscitation 85:657-663

167. Cudnik MT, Sasson C, Rea TD et al (2012) Increasing hospital volume is not associated with improved survival in out of hospital cardiac arrest of cardiac etiology. Resuscitation 83:862-868

168. Heffner AC, Pearson DA, Nussbaum ML, Jones AE (2012) Regionalization of post-cardiac arrest care: implementation of a cardiac resuscitation center. Am Heart J 164:493-501e2

169. Lee SJ, Jeung KW, Lee BK et al (2015) Impact of case volume on outcome and performance of targeted temperature management in out-ofhospital cardiac arrest survivors. Am J Emerg Med 33:31-36

170. Kang MJ, Lee TR, Shin TG et al (2014) Survival and neurologic outcomes of out-of-hospital cardiac arrest patients who were transferred after return of spontaneous circulation for integrated postcardiac arrest syndrome care: the another feasibility of the cardiac arrest center. J Korean Med Sci 29:1301-1307

171. Spiro JR, White S, Quinn N et al (2015) Automated cardiopulmonary resuscitation using a loaddistributing band external cardiac support device for in-hospital cardiac arrest: a single centre experience of AutoPulse-CPR. Int J Cardiol 180:714

172. Wagner H, Rundgren M, Hardig BM et al (2013) A structured approach for treatment of prolonged cardiac arrest cases in the coronary catheterization laboratory using mechanical chest compressions. Int J Cardiovasc Res 2:4
173. Chan TK (2012) New era of CPR: application of itechnology in resuscitation. Hong Kong J Emerg Med 19(5):305-311

174. Zijlstra JA, Stieglis R, Riedijk F, Smeekes M, van der Worp WE, Koster RW (2014) Local lay rescuers with AEDs, alerted by text messages, contribute to early defibrillation in a Dutch out-of-hospital cardiac arrest dispatch system. Resuscitation 85:1444-1449

175. Ringh M, Fredman D, Nordberg P, Stark T, Hollenberg J (2011) Mobile phone technology identifies and recruits trained citizens to perform CPR on out-of-hospital cardiac arrest victims prior to ambulance arrival. Resuscitation 82:1514-1518

176. Jiang C, Zhao Y, Chen Z, Chen S, Yang X (2010) Improving cardiopulmonary resuscitation in the emergency department by real-time video recording and regular feedback learning. Resuscitation 81:1664-1669

177. Stiell IG, Wells GA, Field BJ et al (1999) Improved out-of-hospital cardiac arrest survival through the inexpensive optimization of an existing defibrillation program: OPALS study phase II. Ontario Prehospital Advanced Life Support. JAMA 281:1175-1181

178. Olasveengen TM, Tomlinson AE, Wik L et al (2007) A failed attempt to improve quality of out-ofhospital CPR through performance evaluation. Prehosp Emerg Care 11:427-433

179. Clarke S, Lyon R, Milligan D, Clegg G (2011) Resuscitation feedback and targeted education improves quality of pre-hospital resuscitation in Scotland. Emerg Med J 28(Suppl 1):A6

180. Fletcher D, Galloway R, Chamberlain D, Pateman J, Bryant G, Newcombe RG (2008) Basics in advanced life support: a role for download audit and metronomes. Resuscitation 78:127-134

181. Rittenberger JC, Guyette FX, Tisherman SA, DeVita MA, Alvarez RJ, Callaway CW (2008) Outcomes of a hospital-wide plan to improve care of comatose survivors of cardiac arrest. Resuscitation 79:198-204

182. Wolfe H, Zebuhr C, Topjian AA et al (2014) Interdisciplinary ICU cardiac arrest debriefing improves survival outcomes*. Crit Care Med 42:16881695

183. Hillman K, Chen J, Cretikos M et al (2005) Introduction of the medical emergency team (MET) system: a cluster-randomised controlled trial. Lancet 365:2091-2097

184. Buist MD, Moore GE, Bernard SA, Waxman BP, Anderson JN, Nguyen TV (2002) Effects of a medical emergency team on reduction of incidence of and mortality from unexpected cardiac arrests in hospital: preliminary study. BMJ 324:387-390

185. Beitler JR, Link N, Bails DB, Hurdle K, Chong DH (2011) Reduction in hospital-wide mortality after implementation of a rapid response team: a long-term cohort study. Crit Care 15:R269

186. Chan PS, Khalid A, Longmore LS, Berg RA, Kosiborod M, Spertus JA (2008) Hospital-wide code rates and mortality before and after implementation of a rapid response team. JAMA 300:25062513

187. Konrad D, Jaderling G, Bell M, Granath F, Ekbom A, Martling CR (2010) Reducing in-hospital cardiac arrests and hospital mortality by introducing a medical emergency team. Intensive Care Med 36:100-106

188. Lighthall GK, Parast LM, Rapoport L, Wagner TH (2010) Introduction of a rapid response system at a United States veterans affairs hospital reduced cardiac arrests. Anesth Analg 111:679-686
189. Santamaria J, Tobin A, Holmes J (2010) Changing cardiac arrest and hospital mortality rates through a medical emergency team takes time and constant review. Crit Care Med 38:445-450

190. Priestley G, Watson W, Rashidian A et al (2004) Introducing Critical Care Outreach: a ward-randomised trial of phased introduction in a general hospital. Intensive Care Med 30:1398-1404

191. Delasobera BE, Goodwin TL, Strehlow M et al (2010) Evaluating the efficacy of simulators and multimedia for refreshing ACLS skills in India. Resuscitation 81:217-223

192. Meaney PA, Sutton RM, Tsima B et al (2012) Training hospital providers in basic CPR skills in Botswana: acquisition, retention and impact of novel training techniques. Resuscitation 83:14841490

193. Jain A, Agarwal R, Chawla D, Paul V, Deorari A (2010) Tele-education vs classroom training of neonatal resuscitation: a randomized trial. J Perinatol 30:773-779

194. Jenko M, Frangez M, Manohin A (2012) Four-stage teaching technique and chest compression performance of medical students compared to conventional technique. Croat Med J 53:486-495

195. Li Q, Ma EL, Liu J, Fang LQ, Xia T (2011) Pre-training evaluation and feedback improve medical students' skills in basic life support. Med Teach 33:e549-e555

196. Nilsson C, Sorensen BL, Sorensen JL (2014) Comparing hands-on and video training for postpartum hemorrhage management. Acta Obstet Gynecol Scand 93:517-520

197. Shavit I, Peled S, Steiner IP et al (2010) Comparison of outcomes of two skills-teaching methods on lay-rescuers' acquisition of infant basic life support skills. Acad Emerg Med 17:979-986

198. Bossaert L, Perkins GD, Askitopoulou $\mathrm{H}$ et al (2015) European Resuscitation Council Guidelines for Resuscitation 2015 Section 11 The Ethics of Resuscitation and End-of-Life Decisions. Resuscitation. http://dx.doi.org/10.1016/j.resuscitation.2015.07.033

199. Zideman DA, De Buck EDJ, Singletary EM et al (2015) European Resuscitation Council Guidelines for Resuscitation 2015 Section 9 First Aid. Resuscitation. http://dx.doi.org/10.1016/j.resuscitation.2015.07.031

200. Soar J, Nolan JP, Bottiger BW et al (2015) European Resuscitation Council Guidelines for Resuscitation 2015 Section 3 Adult Advanced Life Support. Resuscitation. http://dx.doi.org/ 10.1016/j.resuscitation.2015.07.016

201. ILCOR Scientific Evidence Evaluation and Review System. https://volunteer.heart.org/apps/pico/ Pages/default.aspx, https://volunteer.heart.org/ apps/pico/Pages/default.aspx. Zugegriffen:10. Mai 2015

202. Sandroni C, Fenici P, Cavallaro F, Bocci MG, Scapigliati A, Antonelli M (2005) Haemodynamic effects of mental stress during cardiac arrest simulation testing on advanced life support courses. Resuscitation 66:39-44

203. Perkins GD, Barrett H, Bullock I et al (2005) The Acute Care Undergraduate TEaching (ACUTE) Initiative: consensus development of core competencies in acute care for undergraduates in the United Kingdom. Intensive Care Med 31:16271633

204. DeVita MA, Smith GB, Adam SK et al (2010) „Identifying the hospitalised patient in crisis" - a consensus conference on the afferent limb of rapid response systems. Resuscitation 81:375-382 


\section{ERC Leitlinien}

205. Smith GB, Osgood VM, Crane S (2002) ALERT-a multiprofessional training course in the care of the acutely ill adult patient. Resuscitation 52:281-286

206. Ringsted C, Lippert F, Hesselfeldt R et al (2007) Assessment of Advanced Life Support competence when combining different test methodsreliability and validity. Resuscitation 75:153-160

207. Perkins GD, Davies RP, Stallard N, Bullock I, Stevens $H$, Lockey A (2007) Advanced life support cardiac arrest scenario test evaluation. Resuscitation 75:484-490

208. Soar J, Perkins GD, Harris S et al (2003) The immediate life support course. Resuscitation 57:21-26

209. Soar J, McKay U (1998) A revised role for the hospital cardiac arrest team? Resuscitation 38:145149

210. Spearpoint KG, Gruber PC, Brett SJ (2009) Impact of the Immediate Life Support course on the incidence and outcome of in-hospital cardiac arrest calls: an observational study over 6 years. Resuscitation 80:638-643

211. Nolan J (2001) Advanced life support training. Resuscitation 50:9-11

212. Perkins G, Lockey A (2002) The advanced life support provider course. BMJ 325:S81

213. Tinsey V (2003) A personal reflection and account on the newborn life support course. MIDIRS Midwifery Digest 13:235-237

214. Singh J, Santosh S, Wyllie JP, Mellon A (2006) Effects of a course in neonatal resuscitation-evaluation of an educational intervention on the standard of neonatal resuscitation. Resuscitation 68:385-389

215. Carapiet D, Fraser J, Wade A, Buss PW, Bingham R (2001) Changes in paediatric resuscitation knowledge among doctors. Arch Dis Child 84:412-414

216. Schebesta K, Rossler B, Kimberger O, Hupfl M (2012) Impact of the European Paediatric Life Support course on knowledge of resuscitation guidelines among Austrian emergency care providers. Minerva anestesiol 78:434-441

217. Cheron G, Jais JP, Cojocaru B, Parez N, Biarent D (2011) The European Paediatric Life Support course improves assessment and care of dehydrated children in the emergency department. Eur J Pediatr 170:1151-1157

218. Charalampopoulos D, Karlis G, Barouxis D et al (2014) Theoretical knowledge and skill retention 4 months after a European Paediatric Life Support course. Eur J Emerg Med (Epub ahead of print)

219. Kirkpatrick D, Kirkpatrick J (2007) Implementing the four levels: a practical guide for the evaluation of training programs. Berrett-Koehler, San Francisco, S 153. ISBN: 978-1-57675-454-2

220. Ringsted C, Hodges B, Scherpbier A (2011), The research compass': an introduction to research in medical education: AMEE Guide no. 56. Med Teach 33:695-709 